\title{
On the definition and examples of Finsler metrics
}

\author{
Miguel Angel Javaloyes and Miguel SÁnchez
}

To Manuel Barros and Angel Ferrández on their $60^{\text {th }}$ birthday

\begin{abstract}
For a standard Finsler metric $F$ on a manifold $M$, the domain is the whole tangent bundle $T M$ and the fundamental tensor $g$ is positive-definite. However, in many cases (for example, for the well-known Kropina and Matsumoto metrics), these two conditions hold in a relaxed form only, namely one has either a pseudo-Finsler metric (with arbitrary $g$ ) or a conic Finsler metric (with domain a "conic" open domain of $T M$ ).

Our aim is twofold. First, we want to give an account of quite a few subtleties that appear under such generalizations, say, for conic pseudo-Finsler metrics (including, as a preliminary step, the case of Minkowski conic pseudo-norms on an affine space). Second, we aim to provide some criteria that determine when a pseudo-Finsler metric $F$ obtained as a general homogeneous combination of Finsler metrics and one-forms is again a Finsler metric - or, more precisely, that the conic domain on which $g$ remains positive-definite. Such a combination generalizes the known $(\alpha, \beta)$-metrics in different directions. Remarkably, classical examples of Finsler metrics are reobtained and extended, with explicit computations of their fundamental tensors.
\end{abstract}

Mathematics Subject Classification (2010): 53C60 (primary); 53C22 (secondary).

\section{Introduction}

Finsler Geometry is a classical branch of Differential Geometry, with a wellestablished background. However, its applications to Physics and other natural sciences (see, for example, $[3,4,27,39,49]$ ) introduced some ambiguity in what is understood as a Finsler manifold. The standard definition of a Finsler metric $F$ on a manifold $M$ entails that $F$ is defined on the whole tangent bundle TM

Both authors are partially supported by Regional J. Andalucía Grant P09-FQM-4496 with FEDER funds. MAJ is also partially supported by MICINN-FEDER project MTM2009-10418 and Fundación Séneca project 04540/GERM/06, and MS by MICINN-FEDER Grant MTM2010-18099.

Received March 7, 2012; accepted in revised form August 14, 2012. 
and that strong convexity is satisfied, i.e., its fundamental tensor $g$ is positive definite $[1,2,5,12,34,44,45]$. In fact, the non-degeneracy of $g$ is essential for many purposes which concern connections and curvature, and its positive-definiteness implies the fundamental inequality, with important consequences on the associated Finsler distance, minimizing properties of geodesics, etc.

However, in many interesting cases, the metric $F$ is defined only in some conic domain $A \varsubsetneqq T M$; recall, for example, the Matsumoto [27] or Kropina metrics [20,24,47], and some metrics constructed as in the Randers case [5, Chapter 11] (see also Corollary 4.17 below). Typically, this happens when $F$ is not positivedefinite or smoothly inextendible in some directions, so that these directions must be removed from the domain of $F$. Many references consider as the essential ingredient of a Finsler metric $F$ that it behaves as a (positively homogeneous) pointwise norm in some conic open subset $A \subset T M[3,4,7,10,25]$. In this case, the role of the positive-definiteness of $g$ may remain somewhat vague. In principle, one admits the pseudo-Finsler case when $g$ is allowed to be non-positive definite. If the nondegeneracy of $g$ is required, then one can redefine $A$ so that degenerate directions are also suppressed. If, moreover, positive-definiteness is required, $A$ will contain only those directions where this property holds. However, notice that it is important then to have criteria which determine exactly the domain $A$, as well as the expected properties for $F$ and $g$ in each case.

The first aim of this work is to study these cases by making two basic extensions of the standard notion of Finsler metric: pseudo-Finsler metrics (if one admits that its fundamental tensor $g$ can be non-positive definite) and conic Finsler metrics (if one admits that the domain of $F$ is not necessarily the whole $T M$ but an open conic domain); if both extensions are done at the same time we speak of a conic pseudo-Finsler metric. Once the general properties of these extensions are studied, our next aim is to determine the regions where strong convexity holds for natural classes of conic pseudo-Finsler metric. Recall that one of the distinctive aspects of Finsler geometry, in comparison with the Riemannian one, is how Finsler metrics (and one-forms) can be combined to obtain new Finsler metrics. In Riemannian geometry the natural operations are just the addition and conformal changes. But in Finsler geometry there is a big amount of possibilities providing endless families of Finsler metrics (see Section 4). As a remarkable goal, we compute the fundamental tensor $g$ of these combinations explicitly, in such a way that the domain where $g$ is positive definite becomes apparent. The paper is divided then in three sections.

In Section 2, our study starts at the very beginning, by discussing accordingly the extensions of Minkowski norms into Minkowski pseudo-norms and Minkowski conic norms - or, in general, Minkowski conic pseudo-norms. After a preliminary review of properties of Minkowski norms in the first subsection, in the second one we focus in their generalizations. As the weakening of these properties may yield the loss of the triangle inequality, we study at the same time norms and norms which satisfy the strict triangle inequality (including a discussion about the weakening of differentiability in Proposition 2.11, Remark 2.12). In the third subsection we show how all these norm-related notions are characterized by looking at the corresponding unit (conic) sphere or indicatrix $S$, which allows one to reconstruct 
the norm from suitable candidates to unit (conic) ball $B$ (Proposition 2.13, Theorem 2.14). The interplay between the convexities of $S, B$ and the conic domain $A$ of the Minkowski conic pseudo-norm is stressed. Finally, in the fourth subsection, simple examples (constructed on $\mathbb{R}^{2}$ from a curve which plays the role of $S$ ) show the optimality of the results. We stress in both, results and examples, that even though the triangle inequality may not hold for Minkowski pseudo-norms, their forward (respectively backward) affine balls still constitute a basis of the topology, as a difference with the conic case (Proposition 2.16). However, for conic Minkowski norms, the sets of all the forward and backward affine balls generate the topology as a subbasis (Proposition 2.8), and suitable triangle inequalities occur when the conic domain $A$ is convex.

In Section 3, the general properties of a conic pseudo-Finsler metric $F$, as well as those for the particular pseudo-Finsler and conic Finsler cases, are studied. It is divided into five subsections with, respectively, the following aims: (1) A discussion on the general definition, including the subtleties inherent to our general choice of conic domain $A \subset T M$ and the possibility of extending it to all $T M$. (2) To introduce the notion of admissible curve (with velocity in $A$ ) and, associated to it: the conic partial ordering $\prec$, the Finsler separation $d_{F}$ (which extends the classical Finsler distance), and the open forward and backward balls, which are shown to be always topologically open subsets but, in general, they do not constitute a basis for the topology (in fact, $d_{F}$ may be identically zero in the conic case). So, the condition of being Riemannianly lower bounded for $F$ is introduced to guarantee that the forward (respectively backward) balls give a basis of the topology. (3) A discussion on the role of the open balls in this general framework. In particular, we show that, in the pseudo-Finsler case, the open forward balls still constitute a basis of the topology, and the Finsler separation $d_{F}$ is still a generalized distance. However, we stress that the corresponding $d_{F}$-balls (obtained as a length space) may look very different from the Finsler case - in particular, they differ from the affine balls for Minkowski pseudo-norms whenever its fundamental tensor $g$ is not positive semidefinite. (4) To introduce geodesics as critical points of the energy functional for admissible curves joining two fixed points. In the non-degenerate directions they are related to the Chern connection, and characterized by means of the geodesic equation (univocally determined from their velocity at some point); otherwise, their possible lack of uniqueness becomes apparent (see Example 2.21). Their minimization properties only appear in the conic Finsler case, and some subtleties about them are especially discussed. (5) A final summary is provided, making a comparison between the Finsler, pseudo-Finsler and conic Finsler cases at three levels: (i) Finsler separation $d_{F}$, (ii) geodesics, and (iii) the particular affine Minkowski case.

In Section 4, we study a general homogeneous functional combination of $n$ (conic) Finsler metrics $F_{1}, \ldots, F_{n}$ and $m$ one-forms $\beta_{n+1}, \ldots, \beta_{n+m}$. Such a $\left(F_{1}, \ldots, F_{n}, \beta_{n+1}, \ldots, \beta_{n+m}\right)$-metrics constitute an obvious generalization of the known $(\alpha, \beta)$-metrics (see [24] and also [18,23,26,29-31,49]) and $\beta$-deformations [40]. In the first subsection, we compute explicitly its fundamental tensor $g$ and derive general conditions to ensure that $g$ is positive definite in Theorem 4.1. Then, some simple particular cases (Corollaries 4.3 and 4.4) and consequences (Remark 
4.5 and Corollary 4.6) are stressed. In the second subsection we focus on the simple case $n=1=m$. The metric $F$ obtained in this case coincides with the so-called $\beta$ deformation of a preexisting Finsler metric $F_{0}$, and it is called here a $\left(F_{0}, \beta\right)$-metric. In particular, classical Matsumoto, Randers and Kropina metrics are reobtained and extended, including the explicit computation of their fundamental tensor $g$, as well as the conic domain for its positive-definiteness. As an example of the possibilities of our approach, we conclude with a further extension to metrics constructed from a pair $\left(F_{1}, F_{2}\right)$ of Finsler ones. Finally, as an Appendix, in the third subsection, our computations are compared with those by Chern and Shen [12] for $(\alpha, \beta)$-metrics.

Finally, we would like to emphasize that, even though our study is quite extensive, it is not by any means exhaustive. There are still natural questions related to the conic pseudo-Finsler metrics which have not been well studied yet and, as some of the ones studied in this paper, may have a non-trivial and subtle answer. Due to the increasing activity in this field, we encourage the readers to make further developments.

ACKNOWLEDgements. The authors warmly acknowledge Professors R. Bryant, E. Caponio and G. Siciliano for helpful conversations on the topics of this paper and the anonimous referee for his interesting comments.

\section{Generalizing Minkowski norms}

\subsection{Classical notions}

First, let us recall the following classical concepts.

Definition 2.1. Let $V$ be a real vector space of finite dimension $N \in \mathbb{N}$ and consider a function $\|\cdot\|: V \rightarrow \mathbb{R}$ being

(i) positive: $\|v\| \geq 0$, with equality if only if $v=0$,

(ii) positively homogeneous: $\|\lambda v\|=\lambda\|v\|$ for all $\lambda>0$.

Then, $\|\cdot\|$ is called:

(a) A positively homogeneous norm, or simply norm, if it satisfies the triangle inequality, i.e.: $\|v+w\| \leq\|v\|+\|w\|$;

(b) A norm with strict triangle inequality, if the equality in the triangle inequality is satisfied only if $v=\lambda w$ or $w=\lambda v$ for some $\lambda \geq 0$;

(c) A Minkowski norm, if: (c1) $\|\cdot\|$ is smooth ${ }^{1}$ away from 0 , so that the fundamental tensor field $g$ of $\|\cdot\|$ on $V \backslash\{0\}$ can be defined as the Hessian of $\frac{1}{2}\|\cdot\|^{2}$, and (c2) $g$ is pointwise positive-definite.

${ }^{1}$ For simplicity, "smooth" will mean $C^{\infty}$ even though differentiability $C^{2}$ will be enough for most purposes. 
About the last definition, recall that the Hessian of any smooth function $f$ is written as $\operatorname{Hess} f(X, Y)=X(Y(f))-\nabla_{X}^{0} Y(f)$ for any vector fields $X, Y$, where $\nabla^{0}$ is the natural affine connection of $V$. Moreover, Minkowski norms always satisfy the strict triangle inequality (see for example [5, Theorem 1.2.2]).

Let us collect some properties of the fundamental tensor $g$ that come directly from its definition and the positive homogeneity.

Proposition 2.2. Given a Minkowski norm $\|\cdot\|$ and $v \in V \backslash\{0\}$, the fundamental tensor $g_{v}$ is given as:

$$
g_{v}(u, w):=\left.\frac{\partial^{2}}{\partial t \partial s} G(v+t u+s w)\right|_{t=s=0},
$$

where $u, w \in V$ and $G=\frac{1}{2}\|\cdot\|^{2}$. Moreover, $v \mapsto g_{v}$ is positively homogeneous of degree 0 (that is, $g_{\lambda v}=g_{v}$ for $\lambda>0$ ) and it satisfies

$$
g_{v}(v, v)=\|v\|^{2}, \quad g_{v}(v, w)=\left.\frac{\partial}{\partial s} G(v+s w)\right|_{s=0} .
$$

Moreover, $v$ is $g_{v}$-orthogonal to the unit sphere $S$, and the metric $g$ is positive definite if and only if so is its restriction to $S$.

Any norm can be characterized by its closed unit ball $B=\{x \in V:\|x\| \leq 1\}$ and its unit sphere or indicatrix $S=\{x \in V:\|x\|=1\}$. For the next result, recall that $V$ is endowed with a natural affine connection $\nabla^{0}$ (as so is any vector or affine space) and, then, any smooth hypersurface admits a second fundamental form $\sigma^{\xi}$ for each transverse $e^{2}$ vector field $\xi$ (see (2.3) below).

Proposition 2.3. Let $\|\cdot\|: V \rightarrow \mathbb{R}$ be a norm and $B$ be its closed unit ball. Then:

(i) Vector 0 belongs to the interior of $B, 0 \in \stackrel{\circ}{B}$;

(ii) $B$ is compact;

(iii) $B$ is convex (i.e., $u, v \in B$ implies $\lambda u+(1-\lambda) v \in B$, for all $\lambda \in[0,1])$.

Moreover:

(iv) The norm $\|\cdot\|$ satisfies the strict triangle inequality iff $B$ is strictly convex (i.e. $u, v \in B$, with $u \neq v$, implies $\lambda u+(1-\lambda) v \in \stackrel{\circ}{B}$, for all $\lambda \in(0,1))$;

(v) The norm $\|\cdot\|$ is a Minkowski one iff $S=\partial B$ is a smooth hypersurface embedded in $V$ and it is strongly convex (i.e. the second fundamental form $\sigma \xi$ of $S$ with respect to some, and then any, transverse vector $\xi$ pointing out to $\stackrel{\circ}{B}$ is positive definite).

2 Even though a Minkowski norm would allow one to define locally a normal direction to the hypersurface (recall for example [52, Definition 3.2.2, Corollary 3.2.3]), this will not play any role here. 
Proof. Standard arguments as in [5,52] are used. Namely, assertions in (i) and (ii) follow from the fact that all the norms on $V$ are equivalent [52, page 29], and (iii), as well as ((iv) $\Rightarrow$ ), is straightforward from the triangle inequality applied to $\lambda u+(1-\lambda) v$. For ((iv) $\Leftarrow)$, in the non-trivial case when $\{u, v\}$ is linearly independent, put $\tilde{u}=u /\|u\|, \tilde{v}=v /\|v\| \in \partial B$ so that

$$
z=\frac{\|u\|}{\|u\|+\|v\|} \tilde{u}+\frac{\|v\|}{\|u\|+\|v\|} \tilde{v} \in \stackrel{\circ}{B} .
$$

Then, $1>\|z\|=\|u+v\| /(\|u\|+\|v\|)$, as required.

For $((v) \Rightarrow)$, recall that positive homogeneity implies that 1 must be a regular value of $\|\cdot\|$ and, so, $S$ is a closed smooth hypersurface in $V$. Moreover, the opposite $\xi$ of the vector position is transverse to $S$ and points out to $\stackrel{\circ}{B}$. So, for any vector fields $X, Y$ tangent to $S$, the decomposition of the canonical connection $\nabla^{0}$ on $V$,

$$
\nabla_{X}^{0} Y=\nabla_{X}^{\xi} Y+\sigma^{\xi}(X, Y) \xi
$$

holds under standard conventions. Putting again $G=\frac{1}{2}\|\cdot\|^{2}$, then

$$
g(X, Y)=\operatorname{Hess} G(X, Y)=-\nabla_{X}^{0} Y(G)
$$

and using (2.3),

$$
g(X, X)=-\nabla_{X}^{\xi} X(G)-\sigma^{\xi}(X, X) \xi(G)=-\sigma^{\xi}(X, X) \xi(G) .
$$

As positive homogeneity implies that $-\xi(G)>0$, we have that $g(X, X)>0$ iff $\sigma^{\xi}(X, X)>0$. For $((\mathrm{v}) \Leftarrow)$, by last statement in Proposition 2.2, we only have to prove that the restriction of $g$ to the indicatrix $S$ is positive definite. Moreover, notice that the positive homogeneity implies that $-\xi$ is transverse and $\|\cdot\|$ is smooth away from 0 . So, (2.5) can be applied again and we are done.

\subsection{Generalized notions}

Next, let us generalize the notion of Minkowski norm.

Definition 2.4. A Minkowski pseudo-norm on $V$ is a map $\|\cdot\|: V \rightarrow \mathbb{R}$ which satisfies (i), (ii) and (c1) in Definition 2.1 (i.e., $g$ is not necessarily positive definite). A Minkowski conic norm on $V$ is a map $\|\cdot\|: A \rightarrow \mathbb{R}$, where $A \subset V$ is a conic domain (i.e., $A$ is open, non-empty and satisfies that if $v \in A$, then $\lambda v \in A$ for all $\lambda>0$ ), which satisfies the conditions (i), (ii), (c1) and (c2) in Definition 2.1 for all $v \in A$ (i.e. $g$ is positive definite, but the conic domain $A$ may not be all $V$ and, in this case, it excludes vector 0 ).

A Minkowski conic pseudo-norm on $V$ is a map $\|\cdot\|: A \rightarrow \mathbb{R}$, which satisfies (i), (ii) and (c1) in Definition 2.1 for all $v \in A$, where $A \subset V$ is a conic domain (i.e., the two previous extensions of the notion of Minkowski norm are allowed simultaneously). 
For simplicity, we will assume typically that $A$ is connected. As in the case of norms, we do not assume that $\|\cdot\|$ is reversible, and we can define two types of affine (normed) balls depending on the order we compute the substraction. Namely, for any Minkowski conic pseudo-norm the forward and backward affine open balls of center $v$ and radius $r>0$ are defined, respectively, as

$$
\begin{aligned}
B_{v}^{+}(r) & =\{x \in v+A:\|x-v\|<r\} \\
\text { and } \quad & B_{v}^{-}(r)=\{x \in v-A:\|v-x\|<r\},
\end{aligned}
$$

where $v \pm A:=\{v \pm w: w \in A\}$. In the case that $g$ is not positive-definite, the behavior of these balls may differ dramatically from the behavior of the metric balls obtained from a length space (see Example 2.23 and Remark 3.16 below), even though the continuity of $\|\cdot\|$ allows one to ensure that they are open subsets. For closed balls $\bar{B}_{v}^{ \pm}(r)$, the non-strict inequality $\leq$ is used instead of $<$ in (2.6); recall that these balls are closed in $v \pm A$ but not in $V$ (except if $V=A$ ). For the forward and backward spheres $S_{v}^{ \pm}(r)$, equalities replace the inequalities in (2.6), and the map

$$
\varphi: S_{v}^{+}(r) \rightarrow S_{v}^{-}(r) \quad w \mapsto 2 v-w
$$

is a homeomorphism. As in the case of norms, we will work by simplicity with $B=\bar{B}_{0}^{+}(1)$ and $S=S_{0}^{+}(1)$ (which is the boundary of $B$ in $A$ ). Recall also that $0 \notin A$ (and thus $0 \notin B$ ) except in the case that $A=V$, i.e., when $\|\cdot\|$ is a pseudo-norm.

Remark 2.5. Observe that Proposition 2.2 is extended directly to this case due to its local nature and the positive homogeneity. Moreover, the expressions (2.3) and (2.5) are also directly transplantable to Minkowski conic pseudo-norms.

The following technical properties must be taken into account.

Proposition 2.6. Let $\|\cdot\|: A \rightarrow \mathbb{R}$ be a Minkowski conic pseudo-norm. Then:

(i) The indicatrix $S$ is a hypersurface embedded in A as a closed subset, and the position vector at each point is transverse to $S$;

(ii) If $\|\cdot\|$ is a pseudo-norm $(A=V)$, $S$ is diffeomorphic to a sphere.

Proof. For (i), positive homogeneity implies that the differential of $\|\cdot\|$ does not vanish on the position vector and, so, $S$ is the inverse image of the regular value 1 .

For (ii), consider the norm $\|\cdot\|^{E}$ associated to any auxiliary Euclidean product on $V$, and let $S^{E}$ be its unit sphere, and observe that the map $S^{E} \rightarrow S, v \mapsto v /\|v\|$ and its inverse are smooth (as so is $\|\cdot\|$ ).

Remark 2.7. As in the proof of the previous proposition, when necessary, we will consider an auxiliary norm $\|\cdot\|^{E}$ associated to some Euclidean product $g^{E}$ on $V$, and the results obtained will be independent of the choice of $g^{E}$. In particular, $V$ can be regarded as a Riemannian manifold with Levi-Civita connection $\nabla^{0}$, the spheres $S_{v}^{ \pm}(r)$ as Riemannian submanifolds with the metric induced by $g^{E}$, and the map (2.7) as an isometry. The $\|\cdot\|^{E}$-open balls will be denoted with a superscript $E$, say as in $B_{v}^{E}(r)$. 
Now, let us focus on Minkowski conic norms. First, we will show that the forward and backward open balls constitute a subbasis for the topology (to check the optimality of this result, see Examples 2.21 and 2.22, and Proposition 2.16 below).

Proposition 2.8. Let $\|\cdot\|: A \rightarrow \mathbb{R}$ be a conic Minkowski norm with A connected. Then, the collection of subsets

$$
\left\{B_{v_{1}}^{+}\left(r_{1}\right) \cap B_{v_{2}}^{-}\left(r_{2}\right): v_{1}, v_{2} \in V, r_{1}, r_{2}>0\right\}
$$

is a topological basis of $V$.

Proof. Using translations, it is enough to show that they yield a topological basis around $0 \in V$.

Choose any $v_{0} \in S$ and an auxiliary Euclidean $g^{E}$ such that $v_{0}$ is also unit and orthogonal to $S$ for $g^{E}$. Let $\theta_{v_{0}}(v)$ be the $g^{E}$-angle between $v \in V \backslash\{0\}$ and $v_{0}$. Fix $\theta_{0} \in(0, \pi / 2)$ satisfying both, every $v \in V$ with $\theta_{v_{0}}(v)<\theta_{0}$ lies in $A$, and for all such $v$ in $S$, the minimum of the $g^{E}$-principal curvatures of $S$ at $v$ (in the inward direction to $B$ ) is greater than some constant $k>0$. Such a constant $k$ exists from the positive definiteness of $g$, putting $\xi$ in (2.5) as the inward $g^{E}$-unit normal (in particular, $\xi_{v_{0}}=-v_{0}$ ). Then, the $g^{E}$-round sphere through $v_{0}$ with radius $r_{k}=1 / k^{2}$ and center $x^{+}$in the $\xi_{v_{0}}$ direction remains outside of $B \cap\left\{v \in V: \theta_{v_{0}}(v)<\theta_{0}\right\}$. Observe that, by the isometry $\varphi$ in (2.7), the analogous properties hold for $-v_{0}$ and $S_{0}^{-}(1)(=\varphi(S))$ with the same value of $k$ (and thus $r_{k}$ ), obtaining then a second center $x^{-}$.

Now, for any neighborhood $U$ of 0 , there exists some $\epsilon>0$ such that $x_{\varepsilon}^{+}=$ $x^{+}-(1-\varepsilon) v_{0}$ and $x_{\varepsilon}^{-}=x^{-}+(1-\varepsilon) v_{0}$ satisfy

$$
B_{x_{\varepsilon}^{+}}^{E}\left(r_{k}\right) \cap B_{x_{\varepsilon}^{-}}^{E}\left(r_{k}\right) \subset U,
$$

and the required property follows because, from the construction,

$$
0 \in B_{(\epsilon-1) v_{0}}^{+}(1) \cap B_{(1-\epsilon) v_{0}}^{-}(1) \subset B_{x_{\varepsilon}^{+}}^{E}\left(r_{k}\right) \cap B_{x_{\varepsilon}^{-}}^{E}\left(r_{k}\right) .
$$

Remark 2.9. We emphasize that in the conic Minkowski case the strict triangle inequality still holds for every $v_{1}, v_{2} \in A$ such that $t v_{1}+(1-t) v_{2} \in A$ for $t \in(0,1)$ (see the proof of parts (iv) and (v) in Proposition 2.3, or Theorem 2.14 below); in particular, it holds for any $v_{1}, v_{2} \in A$ if $A$ is convex. Analogously, if additionally $v_{1} \neq 0$ then $\|\cdot\|$ satisfies the Fundamental Inequality:

$$
\left.\frac{\partial}{\partial t}\left(\left\|v_{1}+t v_{2}\right\|\right)\right|_{t=0} \leq\left\|v_{2}\right\|
$$

or equivalently,

$$
g_{v_{1}}\left(v_{1}, v_{2}\right) \leq\left\|v_{1}\right\|\left\|v_{2}\right\|,
$$


(use Equation (2.2)), where the equality holds if and only if $v_{2}=\lambda v_{1}$ for some $\lambda \geq 0$ (see [12, Section 1.1]).

With more generality, the (strict) triangle inequality for $v_{1}, v_{2} \in A$ in a Minkowski conic pseudo-norm holds when:

(i) $t v_{1}+(1-t) v_{2} \in A$,

(ii) $g$ is positive definite in the direction $t v_{1}+(1-t) v_{2}$,

for every $t \in(0,1)$. As, essentially, the triangle inequality implies the fundamental one (see [5, page 9]), conditions (i) and (ii) are also sufficient to ensure this inequality on $v_{1}$ and $v_{2}$. In particular, if $g_{v_{1}}$ is positive-definite then both, the triangle inequality and the fundamental one, hold in a neighborhood of $v_{1}$. Moreover, these inequalities also hold for any conic Minkowski pseudo-norm under more general hypotheses related to the possible convexity of the indicatrix somewhere. For example, fixing $v_{1} \in A$ the fundamental inequality holds for any $v_{2} \in A$ if the hyperplane $H$ tangent to the indicatrix $S$ at $v_{1} /\left\|v_{1}\right\|$ touches the closed unit ball only at $v_{1}$ (this implies that $g_{v_{1}}$ is positive semi-definite and, even when non-positive definite, the directions $g_{v_{1}}$-orthogonal to $v_{1}$ are those tangent to $H$ ). Indeed, given $v_{1}, v_{2}$, we can assume that $v_{2}=\lambda v_{1}+w$, with $\lambda \geq 0$ and $g_{v_{1}}\left(v_{1}, w\right)=0$ (if $\lambda<0$, the fundamental inequality holds trivially). Observe that the hypothesis on the hyperplane (transplanted to the parallel hyperplane at $\lambda v_{1}$ ) yields $\lambda\left\|v_{1}\right\| \leq\left\|v_{2}\right\|$ with equality only when $\lambda v_{1}=v_{2}$. Then

$$
g_{v_{1}}\left(v_{1}, v_{2}\right)=\lambda\left\|v_{1}\right\|^{2} \leq\left\|v_{1}\right\|\left\|v_{2}\right\|,
$$

and the required fundamental inequality follows.

The word "Minkowski" in Definition 2.4 comprises two properties for the defined objects: (i) they are smooth away from 0 , and (ii) in the case of (conic) norms, the fundamental tensor $g$ is positive definite. Recall, that for a classical norm as in part $(a)$ of Definition 2.1, one has only the weaker properties of continuity and triangle inequality, the former deduced from the latter. This can be extended to the conic case but, as the triangle inequality is involved, the previous remark suggests to impose convexity for the domain $A$ - in particular, $A$ will be connected. Recall that, under this assumption, if there exists a vector $v \in V$ such that $v,-v \in A$ then $0 \in A$ and $A=V$.

Definition 2.10. Let $A$ be a convex conic open subset of $V$. We say that a map $\|\cdot\|: A \rightarrow \mathbb{R}$ satisfying (i) and (ii) in Definition 2.1 for all $v \in A$ is:

(i) a conic norm on $V$ if it satisfies the triangle inequality ( $(a)$ in Definition 2.1),

(ii) a conic norm with strict triangle inequality if it satisfies (b) in Definition 2.1.

Proposition 2.11. Any conic norm $\|\cdot\|: A \rightarrow \mathbb{R}$ is continuous, its open forward and backward balls are open subsets of $V$, and its indicatrix $S$ is a topological hypersurface, which is closed as a subset of A and homeomorphic to an open subset of the usual sphere. 
Proof. Let us show first that the forward and the backward affine balls are open. Given $x \in B_{v}^{+}(r)$, as $A$ is open, we can fix a basis $e_{1}, e_{2}, \ldots, e_{N}$ of $V$ contained in $A$, and such that $z:=x-v=\sum_{i=1}^{N} z^{i} e_{i}$ with $z^{i}>0$ for all $i=1, \ldots, N$. Denote $C=\max \left\{\left\|e_{1}\right\|,\left\|e_{2}\right\|, \ldots,\left\|e_{N}\right\|\right\}$ and $y=\sum_{i=1}^{N} y^{i} e_{i}$ with $y_{i}>0$ for all $i$. By the triangle inequality, if $0<\lambda<1$, the open subset

$$
O_{\lambda}=\left\{v+\lambda(x-v)+y: C \sum_{i=i}^{N} y^{i}<r-\lambda\|x-v\|\right\}
$$

is contained in $B_{v}^{+}(r)$. Moreover, $x \in O_{\lambda}$ when $(1-\lambda) z$ can be chosen as one such $y$, i.e., whenever

$$
(1-\lambda) C \sum_{i=1}^{N} z^{i}<r-\lambda\|x-v\| .
$$

This holds for $\lambda$ close to one, as required. The backward case is analogous.

For the continuity, given $x_{0} \in A$ and $0<\varepsilon<1$, let us find an open neighborhood $\Omega \subset A$ of $x_{0}$ such that $\left|\|x\|-\left\|x_{0}\right\|\right|<\varepsilon$ for all $x \in \Omega$. Choose $y_{ \pm}=(1 \pm \delta) x_{0}$ with $\delta=\frac{\varepsilon}{2\left\|x_{0}\right\|}$ and put $\Omega=B_{y_{-}}^{+}(\varepsilon) \cap B_{y_{+}}^{-}(\varepsilon)$. This subset is open by the first part and, if $x \in \Omega$ :

$\|x\| \leq\left\|x-y_{-}\right\|+\left\|y_{-}\right\|<\varepsilon+\left\|x_{0}\right\|, \quad\left\|x_{0}\right\| \leq\left\|y_{+}\right\| \leq\left\|y_{+}-x\right\|+\|x\|<\varepsilon+\|x\|$

as required.

For the last assertion, consider an auxiliary Euclidean norm $\|\cdot\|^{E}$ with unit sphere $S^{E}$ and put $S_{A}^{E}:=S^{E} \cap A$. Then, the map

$$
\rho: S_{A}^{E} \rightarrow S, \quad v \mapsto v /\|v\|,
$$

is a homeomorphism, and the required properties of $S$ follow.

Remark 2.12. In the case of (conic) pseudo-norms (i.e., only (i) and (ii) in Definition 2.1 are fulfilled) the continuity does not follow because there is no triangle inequality. So, $S$ may be not closed in $A$ (nor a topological hypersurface) and its affine open balls may be non-open as subsets of $V$. Throughout this paper all the conic pseudo-norms will be Minkowski, that is, we will assume that they are smooth away from 0 . Nevertheless, we will also discuss next those which satisfy the (strict or not) triangle inequality, even when $g$ is only positive semi-definite and, so, they are not Minkowski conic norms.

\subsection{Characterization through the unit ball}

In analogy to Proposition 2.3, the unit balls can be described as follows: 
Proposition 2.13. Let $\|\cdot\|: A \rightarrow \mathbb{R}$ be a Minkowski conic pseudo-norm. Then:

(a1) $B$ is a closed subset of $A$ which intersects all the directions $D_{v}:=\{\lambda v: \lambda>$ $0\}, v \in A$;

(a2) $B$ is starshaped from the origin, i.e., $v \in B$ implies $\lambda v \in B$ for all $\lambda \in(0,1)$;

(a3) The boundary $S$ of $B$ in $A$ is a smooth hypersurface and a closed subset of $A$ such that the position vector at each $v \in S$ is transversal (not tangent to $S$ );

(a4) For each $v \in B \backslash\{0\}$ there exists a (necessarily unique $)^{3} \lambda>0$ such that $v / \lambda \in S$.

Proof. All the properties are straightforward from the definition of $B$ and $S$ (for (a3), use part (i) of Proposition 2.6).

Conversely, the unit balls characterize the different types of conic pseudo-norms. In fact, the following theorem (which also strengthens Proposition 2.3) gives a very intuitive picture of all the types of Minkowski conic pseudo-norms defined above. Recall that the classic notions of convexity for $B$ (i.e., as a neighborhood) were included in Proposition 2.3, and the notions of convexity for $S$ (its boundary hypersurface) are included in the next theorem.

Theorem 2.14. Let $A$ be a conic domain of $V$ and $B$ a subset of $A$ which satisfies all the properties (a1) to (a4) in Proposition 2.13. Then the map

$$
\|\cdot\|_{B}: A \rightarrow \mathbb{R}, \quad v \mapsto \operatorname{Inf}\{\lambda>0: v / \lambda \in B\},
$$

is a Minkowski conic pseudo-norm and its closed unit ball is equal to B. Moreover:

(i) $\|\cdot\|_{B}$ is a Minkowski pseudo-norm iff $S$ is homeomorphic to a sphere. In this case, $0 \in \stackrel{B}{B}$ and $\|\cdot\|_{B}$ is continuous in 0 ;

(ii) $\|\cdot\|_{B}$ is a conic Minkowski norm iff $S$ is strongly convex.

Assume now that $A$ is convex. Then:

(iii) $\|\cdot\|_{B}$ is a conic norm iff $B$ is convex and iff $S$ is convex (its second fundamental form with respect to the inner normal is positive semi-definite);

(iv) $\|\cdot\|_{B}$ is a conic norm with strict triangle inequality iff $B$ is strictly convex and iff $S$ is strictly convex (i.e. the hyperplane tangent to $S$ at each point $v_{0}$ only touches $B$ at $v_{0}$ ).

Proof. The map $\|\cdot\|_{B}$ is well defined from property (a1) (in fact, $\|v\|_{B}$ can be defined as the unique $\lambda$ in (a4) if $v \neq 0$ ). Then, it is positive and positively homogeneous. Its smoothness follows from the smoothness of $S$ and the transversality ensured in (a3), as these conditions characterize when the bijective map

${ }^{3}$ It is obvious that the uniqueness of $\lambda$ follows from the definition of Minkowski conic pseudonorm. However, we point out here that it also follows from the previous three items, to stress the independence of the hypotheses in the next theorem. 
$\mathbb{R}^{+} \times S \rightarrow A \backslash\{0\},(t, v) \mapsto t v$ is a diffeomorphism. Moreover, $B$ is the closed unit ball by construction and (a2).

For (i), the implication to the right follows from part (ii) of Proposition 2.6, and the converse because the compactness of $S$ implies that $A=V$. Therefore, assuming that $S$ is a sphere, the properties (a3) and (a4) (or the diffeomorphism given in the previous paragraph) imply that 0 belongs to the inner domain delimited by $S$. Therefore, $B$ is the inner domain (recall (a2) and (a3)), and $0 \in \stackrel{\circ}{B}$. For the continuity, if $\left\{x_{n}\right\}$ converges to 0 but $\left\|x_{n}\right\|_{B}$ does not, we can assume that, up to a subsequence, $\left\|x_{n}\right\|_{B}$ converges to some $c \in(0,+\infty]$. Then, $x_{n} /\left\|x_{n}\right\|$ also converges to some $y \in S$ up to a subsequence, and $x_{n} \rightarrow c y \neq 0$, which gives a contradiction.

For (ii), recall that positive homogeneity implies that 1 must be a regular value of $\|\cdot\|$ and, so, $S$ is a closed smooth hypersurface in $A$. Moreover, the opposite $\xi$ of the vector position is transverse to $S$ and points out to $\stackrel{\circ}{B}$. Putting $G=\frac{1}{2}\|\cdot\|_{B}^{2}$ and using (2.5) (recall Remark 2.5), $\sigma^{\xi}(X, X)>0$ if and only if $g(X, X)>0$ for every vector field $X$ tangent to $S$. This statement together with last statement in Proposition 2.2 (recall again Remark 2.5) prove (ii).

For (iii) and (iv), even though the equivalences between the (strict or not) convexities of $B$ and $S$ are known (see for example $[6,43]$ and references therein), we will prove the full cyclic implications for the sake of completeness. Moreover, the first implications to the right in (iii) and (iv) are straightforward from the triangle inequality applied to $\lambda u+(1-\lambda) v$. For the second implication to the right, consider a straight line $\mathbf{r}$ tangent to $S$ at some $v_{0} \in S$ with direction $v$, and let $(a, b) \subset \mathbb{R}$ be the interval of points $t \in \mathbb{R}$ such that $v_{0}+t v \in A$ (recall that $A$ is convex). Then the function $f:(a, b) \rightarrow \mathbb{R}$, given by $f(t)=G\left(v_{0}+t v\right)-1$, where $G=\frac{1}{2}\|\cdot\|_{B}^{2}$, satisfies $f(0)=0, \dot{f}(0)=0$ (as $\left.d G_{v_{0}}(v)=0\right)$ and $\sigma_{v_{0}}^{\xi}(v, v)=-\operatorname{Hess}(v, v)=-\ddot{f}(0)$ (recall (2.4) and (2.3) above). Now consider the plane $\pi=\operatorname{span}\left\{v_{0}, v\right\}$. If $\ddot{f}(0)<0$, then the intersection $\pi \cap S$ must lie, close to $v_{0}$, in the connected component determined by the line $\mathbf{r}$ that does not contain 0 . Easily, this contradicts the convexity of $B$ and proves the implication in part (iii). For (iv), if $\mathbf{r}$ touches $\pi \cap S$ in two points, then $S$ must contain a whole segment by convexity and it cannot be strictly convex. Reasoning with all the straight lines tangent to $S$ in $v_{0}$ we conclude that the tangent hyperplane in $v_{0}$ touches only $v_{0}$.

To close the cyclic implications in (iii) and (iv), we have to prove that if $S$ is (strictly) convex, then $\|\cdot\|_{B}$ satisfies the (strict) triangle inequality. Given $u, v \in B$ (linearly independent), consider $\tilde{u}=u /\|u\|_{B}, \tilde{v}=v /\|v\|_{B} \in S$ and define $\phi$ : $[0,1] \rightarrow \mathbb{R}$ as

$$
\phi(t)=G(t \tilde{u}+(1-t) \tilde{v})=G(\tilde{v}+t(\tilde{u}-\tilde{v})) .
$$

If we denote $y=\tilde{v}+t(\tilde{u}-\tilde{v})$, then $\ddot{\phi}(t)=g_{y}(\tilde{u}-\tilde{v}, \tilde{u}-\tilde{v}) \geq 0$ for $t \in(0,1)$ and $\phi(0)=\phi(1)=1$. It is easy to prove that $\phi$ is constantly equal to 1 or $\phi(t)<1$ for $t \in(0,1)$. In particular, for $t=\|u\|_{B} /\left(\|u\|_{B}+\|v\|_{B}\right)$ we obtain

$$
z=\frac{\|u\|_{B}}{\|u\|_{B}+\|v\|_{B}} \tilde{u}+\frac{\|v\|_{B}}{\|u\|_{B}+\|v\|_{B}} \tilde{v} \in B .
$$


Then, $1 \geq\|z\|_{B}=\|u+v\|_{B} /\left(\|u\|_{B}+\|v\|_{B}\right)$, as required. Regarding the strict inequality, observe that if $S$ is strictly convex, $\phi$ cannot be constantly equal to 1 .

Remark 2.15. In the previous theorem, if the hypothesis (a4) of Proposition 2.13 were not imposed, then $\|\cdot\|_{B}$ would not be positive. That is, $\|v\|_{B} \geq 0$ would still hold for all $v \in A$, but the equality could be reached for some $v \neq 0$ and, thus, for all its degenerate direction $D_{v}$. This could happen even if $A=V$ and, in this case, $B$ would not be compact (nor $S$ homeomorphic to a sphere). Even though this possibility could be also admitted, we prefer not to include it. Or, equally, if degenerate directions appear, $A$ is supposed to be redefined in order to exclude them.

Finally, we consider in particular the case of pseudo-norms.

Proposition 2.16. Let $\|\cdot\|: V \rightarrow \mathbb{R}$ be a Minkowski pseudo-norm. Then:

(i) The affine open forward (respectively backward) balls constitute a basis for the natural topology of $V$;

(ii) If $\|\cdot\|$ is not a Minkowski norm, the fundamental tensor field $g$ is degenerate at some direction.

Proof. Choose an auxiliary Euclidean norm $\|\cdot\|^{E}$ and denote $S^{E}$ its unit sphere.

For (i), the compactness of the unit spheres of $\|\cdot\|^{E}$ and $\|\cdot\|$ implies that each ball for one (pseudo-)norm contains small balls for the other one.

For (ii), it is well-known that, at any point $v_{0}$ in $S$ which is a relative maximum for the $\|\cdot\|^{E}$-distance, the second fundamental form $\sigma^{\xi}$ with respect to $\xi=-v_{0}$ must be positive definite (see [19, Chapter VII, Proposition 4.6]). Thus, so is $g$ at $v_{0}$ (recall Equation (2.5) and Remark 2.5 together with last statement of Proposition 2.2) and, as we assume that $\|\cdot\|$ is not a Minkowski norm, there must exist $v_{1} \in V$ where $g$ is degenerate (see also [22]).

Remark 2.17. These two properties cannot be extended to Minkowski conic norms - this is trivial for (ii), and see Example 2.22 below for (i).

\subsection{Some simple examples}

Theorem 2.14 provides a simple picture of Minkowski norms and their generalizations in terms of the closed unit ball $B$ and the indicatrix $S$. Next, we consider $\mathbb{R}^{2}$ and construct illustrative examples of the generalizations of Minkowski norms stated above - easily, these examples can be extended to higher dimensional vector spaces.

Consider a curve $c: I \subset \mathbb{R} \rightarrow \mathbb{R}^{2}, c(\theta)=r(\theta)(\cos \theta$, $\sin \theta)$, which does not cross the origin and is smoothly parameterized by its angular polar coordinate $\theta \in I$. For each $\theta \in \mathbb{R}$ consider the radial half line

$$
l_{\theta}=\{r(\cos \theta, \sin \theta): r>0\}
$$


Recall that $c$ crosses transversally all $l_{\theta}$ with $\theta \in I$, and let $n(\theta)$ be the normal vector characterized by $\langle n(\theta), \dot{c}(\theta)\rangle_{0}=0,-\langle n(\theta), c(\theta)\rangle_{0}>0$, where $\langle\cdot, \cdot\rangle_{0}$ is the natural scalar product on $\mathbb{R}^{2}$. Put also $S=c(I)$ and

$$
\hat{g}(\theta)=\langle\ddot{c}(\theta), n(\theta)\rangle_{0}=\frac{2 \dot{r}^{2}+r(r-\ddot{r})}{\sqrt{r^{2}+\dot{r}^{2}}} .
$$

Recall that the sign of $\hat{g}(\theta)$ is equal to the sign of the second fundamental form of $S$ at $c(\theta)$ with respect to the direction $\xi=-c(\theta)$, and it is also equal to the sign of the fundamental tensor field $g$ on the non-zero vectors of $T_{c(\theta)} S$.

Let us then construct some examples.

Example 2.18. Assume that $c$ is a smooth closed curve, say, $I=[0,2 \pi]$, and $c$, as well as all its derivatives, agree at 0 and at $2 \pi$. By Theorem 2.14 the interior region $B$ of $S$ is the unit ball of a Minkowski pseudo-norm $\|\cdot\|_{B}$. Clearly $\hat{g}(\theta) \geq 0$ everywhere iff $S$ is convex and $\|\cdot\|_{B}$ becomes a norm; in this case, if $\hat{g}(\theta)$ is equal to zero only at isolated points then $S$ is strictly convex and $\|\cdot\|_{B}$ satisfies the strict triangle inequality. The strict inequality $\hat{g}>0$ characterizes when $\|\cdot\|_{B}$ is a Minkowski norm.

Example 2.19. Assume that $I=\left(\theta_{-}, \theta_{+}\right)$with $\theta_{+}-\theta_{-}<\pi$. Now, $A=\cup_{\theta \in I} l_{\theta}$ constitutes a convex conic domain of $\mathbb{R}^{2}$, and take the closed subset $B$ of $A$ delimited by $l_{\theta_{-}}, l_{\theta_{+}}$and $S$. Again, $B$ is the closed unit ball for a Minkowski conic pseudo-norm $\|\cdot\|_{B}$, which will be a Minkowski conic norm iff $\hat{g}>0$ (recall that all the items of Theorem 2.14 will be applicable).

If $c$ admits a smooth extension to $\theta_{-}$or $\theta_{+}$which is transverse to the corresponding $l_{\theta}$, then $\|\cdot\|_{B}$ can be regarded as the restriction to $A$ of a Minkowski conic pseudo-norm defined on a bigger domain $\tilde{A}$. Recall that, if $S$ is convex, $c$ can always be extended in $\mathbb{R}^{2}$ to either $\theta_{-}$or $\theta_{+}$as the tangent line at any $\theta_{0} \in I$ must intersect either $l_{\theta_{-}}$or $l_{\theta_{+}}$, but this extension may be non-transverse, or even the point zero (put $I=(0, \pi / 2)$ and choose $c$ as the $\theta$-reparametrization of the branch $x>x_{0}$ of the parabola $y=\left(x-x_{0}\right)^{2}$ for some $\left.x_{0} \geq 0\right)$.

Observe that, by construction, $S$ is always closed as a subset of $A$. If $S$ is also closed as a subset of $V$ then $S$ cannot be convex everywhere (essentially, $l_{\theta_{-}}$and $l_{\theta_{+}}$would be parallel to asymptotes of $c$ ). In this case, it would be also natural to extend $\|\cdot\|_{B}$ to all $V$, so that the directions $l_{\theta_{ \pm}}$, away from $A$, were degenerate (see part (1) of Remark 2.15).

Example 2.20. Let $c(\theta)=\theta(\cos \theta, \sin \theta), \theta \in(\epsilon, 2 \pi-\epsilon)$ for some $\epsilon>0$. Clearly, this curve is convex (use (2.9)) and determines a Minkowski conic norm $\|\cdot\|$. For small $\epsilon,\|\cdot\|$ has a non-convex domain $A$ and satisfies: (i) it is not the restriction to $A$ of a Minkowski norm on all $\mathbb{R}^{2}$, and (ii) there are vectors $u, v \in A$ such that $u+v \in$ $A$ but they do not satisfy the triangle inequality (choose $u=(0,2 \sin (2 \varepsilon)), v=$ $(\cos (2 \epsilon),-\sin (2 \varepsilon))$. Then $\|u\|=4 \sin (2 \varepsilon) / \pi,\|v\|=1 /(\pi-2 \varepsilon)$ and $\|u+v\|=$ $1 /(2 \varepsilon)$. It is clear that when $\varepsilon$ is small enough, $\|u\|+\|v\|<\|u+v\|)$.

Example 2.21. Consider now two examples with $A=\left\{(x, y) \in \mathbb{R}^{2}: y>0\right\}$. First, the curve $c_{1}$ obtained as the reparametrization of a branch of the parabola 
$t \mapsto(t, \sqrt{1-t}), t \in(-\infty, 1)$, with the angular coordinate $\theta \in(0, \pi)$. In this case $\hat{g}>0$, the curve defines a Minkowski conic norm and Proposition 2.8 is applicable. Second, the curve $c_{2}$ obtaining as reparametrization of the straight line $y=1$ (i.e., the associated norm is $\|(x, y)\|:=y$ ). This is a conic norm, but the forward and backward balls do not constitute a subbasis for the topology of $\mathbb{R}^{2}$. Moreover, all its admissible curves connecting two fixed points will have the same length and, according to Subsection 3.4, they will be extremal of the energy functional (geodesics) when reparameterized at constant speed.

Example 2.22. As a refinement of the previous example for future referencing, consider the parabola $c(t)=\left(t, 1-t^{2}\right), t \in \mathbb{R}$ which can be reparameterized with the angular coordinate $\theta \in(-\pi / 2,3 \pi / 2)$. This curve is strongly convex and defines a Minkowski conic norm with only a direction excluded from the domain - concretely, $A=\mathbb{R}^{2} \backslash\left(l_{-\pi / 2} \cup\{0\}\right)$. Its affine open balls (see (2.6)) centered at any $v_{0}=\left(x_{0}, y_{0}\right)$ are delimited by some parabola as follows:

$$
B_{v_{0}}^{+}(r)=\left\{(x, y):(x, y)-v_{0} \in A ; y-y_{0}<r\left(1-\frac{\left(x-x_{0}\right)^{2}}{r^{2}}\right)\right\} .
$$

In this example (as well as in the first one in Example 2.21), the balls are always open for the topology of $V$ but, as two such parabolas always intersect, the topology generated by the balls on $\mathbb{R}^{2}$ is strictly coarser than the usual one (in particular, no pair of points are Hausdorff related for that topology). Thus, the result in part (i) of Proposition 2.16 cannot be extended to the case of Minkowski conic norms.

Example 2.23. Consider the curve $c(t)=(\sinh t, \cosh t)$, for $t \in \mathbb{R}$, which can be reparametrized by the angular coordinate $\theta \in(\pi / 4,3 \pi / 4)$ and, thus, can be regarded as a particular case of Example 2.19. This curve defines a conic pseudonorm $\|\cdot\|$ with $S$ concave everywhere $($ i.e. $\hat{g}<0$ ), which can be interpreted as follows.

Consider the natural Lorentzian scalar product on $\mathbb{R}^{2}$ :

$$
\left\langle(x, y),\left(x^{\prime}, y^{\prime}\right)\right\rangle_{1}=x x^{\prime}-y y^{\prime} .
$$

Then, $A$ is composed of all the vectors $v=(x, y)$ which are timelike $\left(\langle(x, y),(x, y)\rangle_{1}<\right.$ $0)$ and future-directed $(y>0)$; moreover:

$$
\|v\|=\sqrt{-\langle(x, y),(x, y)\rangle_{1}} .
$$

Here, the (forward) affine balls centered at the origin can be described as:

$$
B_{0}^{+}(r)=\left\{v \in A:-\langle v, v\rangle_{1}<r^{2}\right\},
$$

which is the open region delimited by a hyperbola and the lines $y= \pm x$.

Remarkable, the triangle inequality does not hold, but a reverse strict triangle inequality does, namely:

$$
\|v+w\| \geq\|v\|+\|w\|, \quad \forall v, w \in A
$$


with equality iff $w=\lambda v$ for some $\lambda>0$ (this and the following property are wellknown for Lorentzian scalar products, see [36, Proposition 5.30], for example). By applying this triangle inequality, the following property follows easily. For all $v_{0} \in A$ and $\epsilon>0$ there exists a sequence $P_{0}=0, P_{1}, \ldots, P_{k}=v_{0}$ such that each $v_{i}=P_{i}-P_{i-1}$ belongs to $A$ and $\sum_{i=1}^{k}\left\|v_{i}\right\|<\epsilon$. That is, there are poligonal curves connecting 0 and $v_{0}$ with arbitrarily short $\|\cdot\|$-length.

Finally, recall also that $\|\cdot\|$ can be naturally extended to past-directed timelike vectors, or even all the vectors where the Lorentzian scalar product does not vanish, yielding a bigger conic domain $\tilde{A}$ with four connected parts. Such a situation becomes natural for 2-homogeneous Finsler manifolds (see part (1) of Remark 3.2 below).

\section{Pseudo-Finsler and conic Finsler metrics}

\subsection{Notion}

First, let us clarify the notions of Finsler metric to be used.

Definition 3.1. Let $M$ be a manifold and $A$ an open subset of the tangent bundle $T M$ such that $\pi(A)=M$, where $\pi: T M \rightarrow M$ is the natural projection, and let $F: A \rightarrow[0, \infty)$ be a continuous function. Assume that $(A, F)$ satisfies:

(i) $A$ is conic in $T M$, i.e., for every $v \in A$ and $\lambda>0, \lambda v \in A-$ or, equivalently, for each $p \in M, A_{p}:=A \cap T_{p} M$ is a conic domain in $T_{p} M$.

(ii) $F$ is smooth on $A$ except at most on the zero vectors.

We say that $(A, F)$, or simply $F$, is a conic pseudo-Finsler metric if each restriction $F_{p}:=\left.F\right|_{A_{p}}$ is a Minkowski conic pseudo-norm on $T_{p} M$. In this case, $F$ is

(iii) a conic Finsler metric if each $F_{p}$ is a Minkowski conic norm, i.e. the fundamental tensor $g$ on $A \backslash$ \{zero section\} induced by all the fundamental tensor fields $g^{(p)}$ at each $p \in M$ on $A_{p} \backslash\{0\}$ is positive definite for all $p \in M$,

(iv) a pseudo-Finsler metric if $A=T M$, i.e., each $F_{p}$ is a Minkowski pseudonorm so that $A_{p}=T_{p} M$ for all $p \in M$,

(v) a (standard) Finsler metric if $F$ is both, conic Finsler and pseudo-Finsler, i.e. $A=T M$ and $g$ is pointwise positive definite.

Observe that the fundamental tensor $g$ can be thought as a section of a fiber bundle over $A$. To be more precise, denote also as $\pi: A \backslash\{0\} \rightarrow M$ the restriction of the natural projection from the tangent bundle to $M, T M^{*}$ the cotangent bundle of $M$ and $\tilde{\pi}: T M^{*} \rightarrow M$ the natural projection. Define $\tilde{\pi}^{*}: \pi^{*}\left(T M^{*}\right) \rightarrow A \backslash\{0\}$ as the fiber bundle obtained as the pulled-back bundle of $\tilde{\pi}: T M^{*} \rightarrow M$ through $\pi: A \backslash\{0\} \rightarrow M:$

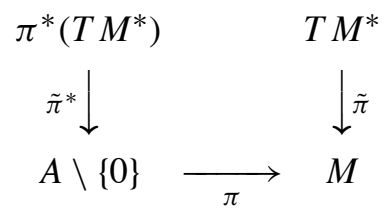


Then $g$ is a smooth symmetric section of the fiber bundle $\pi^{*}\left(T M^{*}\right) \otimes \pi^{*}\left(T M^{*}\right)$ over $A \backslash\{0\}$. Let us remark that if we fix a vector $v \in A \backslash\{0\}$, then $g_{v}$ is a symmetric bilinear form on $T_{\pi(v)} M$. From now on this will be the preferred notation.

Remark 3.2. Some comments are in order:

(1) Our definition of (standard) Finsler metric agrees with classical references as $[1,2,5,12,34,44,45]$ and our definition of conic Finsler metric is equivalent to the notion of generalized Finsler metric by [10] (but we retain our nomenclature as we are also dealing with other generalizations). According to our nomenclature, a classical Kropina metric is a conic Finsler metric (see Corollary 4.12 below). A Matsumoto metric has a maximal conic domain where it is conic Finsler and a bigger one where it is conic pseudo-Finsler (see Corollary 4.15 below). Another way to generalize Finsler metrics is considering what we would call a 2-homogeneous Finsler metric $L$ (here $L$ would be positively homogeneous of degree 2 , and the definition would include all the Lorentzian or semi-Riemannian metrics). This approach can be found in $[3,4,25,37]$. In principle, the case of conic 2-homogeneous Finsler metrics is more general as one can always consider the conic 2-homogeneous Finsler metric $L=F^{2}$ associated to any conic pseudo-Finsler metric. The converse holds only when $L$ is positive away from the zero section (otherwise, one can define just a conic pseudo-Finsler metric on the positive-definite directions). Nevertheless, it is natural to assume then some minimum restrictions which essentially reduces to our case ${ }^{4}$. So, we will focus on the already general conic pseudo-Finsler case, which allows one to clarify some properties of the distance and balls. Let us finally point out that, in reference [7], the authors define a Finsler metric as what we call a conic Finsler metric and a pseudo-Finsler metric as a conic 2-homogeneous Finsler metric with nondegenerate fundamental tensor.

(2) Minkowski conic pseudo-norms as those studied in Subsection 2.4 give the first examples of conic pseudo-Finsler metrics. Starting at them, one can yield examples of more general situations. For instance, consider the following two conic pseudo Finsler metrics on $\mathbb{R}^{2}$ :

(a) $A=\left\{v \in T \mathbb{R}^{2}: d y(v)>0\right\}$ and $F=\left.d y\right|_{A}$,

(b) $A=\left\{v \in T \mathbb{R}^{2}: d x(v) \neq 0, d y(v) \neq 0\right\}$ and $F=\sqrt{d x^{2}}+\sqrt{d y^{2}}$.

Recall that they come from Minkowski conic pseudo-norms with non-compact and convex indicatrices, which are not strongly convex at any point (so that only the non-strict triangle inequality will hold). It is not difficult to accept that a conic pseudo-Finsler metric $F^{a}$ on $\mathbb{R}^{2}$ can be defined such that $F_{(x, y)}^{a}$ behaves as the metric in item (a) for $|y|<1$, as the conic Minkowski norm in Example 2.22 for $y<-2$ and as the Minkowski pseudo-norm in the Example 2.23 for $y>2$. Analogously, a Finsler metric $F^{b}$ on $\mathbb{R}^{2}$ can be defined such

4 See, for example, the definition of Finsler spacetime in [38]. Its associated Finsler metric satisfies all the assumptions of a conic pseudo-Finsler metric (except strict positive definiteness) plus other additional hypotheses. 
that $F_{(x, y)}^{b}$ behaves as the metric in (b) for $x^{2}+y^{2}<1$ and as the Finsler metric associated to the usual scalar product on $\mathbb{R}^{2}$ for $x^{2}+y^{2}>2$. In particular, the conic domain $A_{p}$ may vary from point to point so that such domains are not homeomorphic.

(3) As the last example shows, the number of connected components of $A_{p}$ may vary with $p$, and $A$ may be connected even if some $A_{p}$ are not. Recall that for a Minkowski conic pseudo-norm $\|\cdot\|$, the conical domain $A$ might have (infinitely) many connected parts, even though we consider typically the case $A$ connected. Accordingly, a natural hypothesis in the conic pseudo-Finsler case is to assume that $A$ is pointwise connected (i.e., any $A_{p}, p \in M$, is connected) and, in the conic Finsler case, we also may assume that $A$ is pointwise convex ( $A_{p}$ is a convex subset, and then connected, of each $T_{p} M$ ) in order to have the triangle inequality.

(4) Typically, one can also assume that the pair $(A, F)$ is maximal, i.e., such that no pair $(\tilde{A}, \tilde{F})$ extends $(A, F)$. This means that no conic pseudo-Finsler met$\operatorname{ric}(\tilde{A}, \tilde{F})$ satisfies $A \varsubsetneqq \tilde{A}$ with $\left.\tilde{F}\right|_{A}=F$ (say, as in Examples 2.22 or 2.23). Zorn's lemma ensures the existence of maximal extensions. However, as such an extension may be highly non-unique (recall Example 2.19 or the first one in Example 2.21), and maximality will not be assumed a priori along this paper. Moreover, non-maximal pairs $(A, F)$ may be useful to model diverse situations - for example, to represent restrictions to the possible velocities on relativistic particles which may move on $M$.

(5) As a direct consequence of part (ii) of Proposition 2.16, the fundamental tensor $g$ of a pseudo-Finsler but not Finsler manifold must be degenerate at some points, concretely, it must be degenerate on some directions at all the tangent spaces $T_{p} M, p \in M$, where $g^{(p)}$ is not definite positive.

Some properties of conic pseudo-Finsler metrics can be reduced to the case $A=$ $T M$ taking into account the following result.

Proposition 3.3. Let $F: A \rightarrow \mathbb{R}$ be a conic pseudo-Finsler metric and let $C \subset A$ be such that $C \cup\{$ zero section $\}$ is a closed conic subset of $T M$. Then, there exists a pseudo-Finsler metric $\tilde{F}$ on $M$ such that $\left.\tilde{F}\right|_{C}=\left.F\right|_{C}$.

Proof. Consider some auxiliary Riemannian metric $g_{R}$ on $M$, let $F_{R}=\sqrt{g_{R}}$ be its associated Finsler metric, and take its unit sphere bundle $S_{R} M \subset T M$. Let $\{U, V\}$ be the open covering of $S_{R} M$ defined as $U=S_{R} M \cap(T M \backslash C)$ and $V=$ $S_{R} M \cap A$. Consider a partition of the unity $\left\{\mu_{U}, \mu_{V}\right\}$ subordinated to this covering, and regard these functions as functions on $T M \backslash\{$ zero section $\}$ just by making them homogeneous of degree 0 . The pseudo-Finsler metric $\tilde{F}=\mu_{U} F_{R}+\mu_{V} F$ satisfies the required properties.

Remark 3.4. Recall that, in the previous proposition, the functions which constitute the partition of the unity are not defined on $M$ but on $T M$. So, when applied to a conic Finsler metric, the obtained extension is only a conic pseudo-Finsler metric (see Example 2.20). 


\subsection{Admissible curves and Finslerian separation}

In this subsection, $M$ will be assumed to be connected in order to avoid trivialities. Consider a smooth curve $\alpha$ with velocity $\dot{\alpha}$ in a conic pseudo-Finsler manifold $(M, F)$. As the expression $F(\dot{\alpha})$ does not always make sense, we need to restrict to curves where it does.

Definition 3.5. Let $F: A \rightarrow[0, \infty)$ be a conic pseudo-Finsler metric on $M$. A piecewise smooth curve $\alpha:[a, b] \rightarrow M$ is $F$-admissible (or simply admissible) if the right and left derivatives $\dot{\alpha}_{+}(t)$ and $\dot{\alpha}_{-}(t)$ belong to $A$ for every $t \in[a, b]$. In this case, the $(F-)$ length of $\alpha$ is defined as

$$
\ell_{F}(\alpha)=\int_{a}^{b} F(\dot{\alpha}(t)) \mathrm{d} s .
$$

Definition 3.6. Let $(M, F)$ be a conic pseudo-Finsler manifold, and $p, q \in M$. We say that $p$ precedes $q$, denoted $p \prec q$, if there exists an admissible curve from $p$ to $q$. Accordingly, the future and the past of $p$ are the subsets

$$
\mathrm{I}^{+}(p)=\{q \in M: p \prec q\} \text { and } \mathrm{I}^{-}(p)=\{q \in M: q \prec p\},
$$

respectively. Moreover, we define $\mathrm{I}^{+}=\{(p, q) \in M \times M: p \prec q\}$, i.e., $\mathrm{I}^{+}$is equal to the binary relation $\prec$ as a point subset of $M \times M$.

It is straightforward to check the following properties:

Proposition 3.7. Given a conic pseudo-Finsler manifold $(M, F)$ :

(i) The binary relation $\prec$ is transitive;

(ii) The subsets $\mathrm{I}^{+}(p)$ and $\mathrm{I}^{-}(p)$ are open in $M$ for all $p \in M$, and $\mathrm{I}^{+}$is open in $M \times M$;

(iii) If $F$ is a pseudo-Finsler metric then $\prec$ is trivial, i.e., $p \prec q$ for all $p, q$ in the (connected) manifold $M$.

(For (ii), recall the techniques in the proof of Proposition 3.9 below.)

Now, we can introduce a generalization of the so-called Finsler distance for Finsler manifolds.

Definition 3.8. Let $(M, F)$ be a conic pseudo-Finsler manifold $p, q \in M$ and $C_{p, q}^{F}$ the set of all the $F$-admissible piecewise smooth curves from $p$ to $q$. The Finslerian separation from $p$ to $q$ is defined as:

$$
\mathrm{d}_{F}(p, q)=\inf _{\alpha \in C_{p, q}^{F}} \ell_{F}(\alpha) \in[0, \infty]
$$

From the definitions, one has directly:

$$
C_{p, q}^{F} \neq \emptyset \Leftrightarrow p \prec q, \quad \mathrm{~d}_{F}(p, q)=\infty \Leftrightarrow p \nprec q, \quad \mathrm{~d}_{F}(p, q) \geq 0,
$$


as well as the triangle inequality

$$
\mathrm{d}_{F}(p, q) \leq \mathrm{d}_{F}(p, z)+\mathrm{d}_{F}(z, q), \quad \forall p, q, z \in M
$$

Analogously, we can define two kinds of balls, the (open) forward $d_{F}$-balls $B_{F}^{+}(p, r)$ and the backward ones $B_{F}^{-}(p, r)$, namely,

$$
B_{F}^{+}(p, r)=\left\{q \in M: \mathrm{d}_{F}(p, q)<r\right\}, \quad B_{F}^{-}(p, r)=\left\{q \in M: \mathrm{d}_{F}(q, p)<r\right\} .
$$

Proposition 3.9. Let $(M, F)$ be a conic pseudo-Finsler manifold. Then the open forward and backward $d_{F}$-balls are open subsets.

Proof. Let $q \in B_{F}^{+}(p, r)$ and $\alpha:\left[0, r_{0}\right] \rightarrow M$ be an admissible curve from $p$ to $q$ parametrized by arclength, so that $r_{0}<r$ and $\dot{\alpha}\left(r_{0}\right)$ belongs to $A \cap T_{q} M$. Let $K$ be a compact neighborhood of $\dot{\alpha}\left(r_{0}\right)$ in the unit ball at $T_{q} M$ entirely contained in $A$, and $C$ the corresponding conical subset $C=\{t v: v \in K, t>0\}$. Choosing coordinates $(U, \varphi)$ in a neighborhood $U$ of $q, T U$ can be written as $\varphi(U) \times \mathbb{R}^{n}$ and $C$ is identified with some $\{\varphi(q)\} \times C_{0}$, namely $C_{0}=\left\{t u: u \in K_{0}, t>0\right\}$ is a conical subset of $\mathbb{R}^{n}$, and the compact subset $K_{0}$ is defined by $d \varphi(K)=\{q\} \times K_{0}$. Moreover, choosing a smaller $U$ if necessary (say, with compact closure), we can assume $\varphi(U) \times C_{0} \subset d \varphi(A)$ and $F \circ \varphi^{-1}$ is bounded on $\varphi(U) \times K_{0}$ by some constant $N_{0} \geq 1$. So, choose some $r_{-}<r_{0}$ with $r_{0}-r_{-}<\left(r-r_{0}\right) / N_{0}$, such that $\alpha\left(\left[r_{-}, r_{0}\right]\right) \subset U$, the segment from $\varphi\left(\alpha\left(r_{-}\right)\right)$to $\varphi\left(\alpha\left(r_{0}\right)\right)$ is contained in $U$ and there exists $\lambda>1$ with $\frac{\lambda}{r_{0}-r_{-}}\left(\varphi\left(\alpha\left(r_{0}\right)\right)-\varphi\left(\alpha\left(r_{-}\right)\right)\right) \in K_{0}$. (Observe that we can always find such an $r_{-}$because

$$
\lim _{r_{-} \rightarrow r_{0}} \frac{\varphi\left(\alpha\left(r_{0}\right)\right)-\varphi\left(\alpha\left(r_{-}\right)\right)}{r_{0}-r_{-}}=d \varphi\left(\dot{\alpha}\left(r_{0}\right)\right)
$$

and $\left.d \varphi\left(\dot{\alpha}\left(r_{0}\right)\right) \in K_{0}\right)$. The segments in $\varphi(U)$ (regarded as a subset of $\mathbb{R}^{n}$ ) which start at $\alpha\left(r_{-}\right)$with velocity in $K_{0}$ and defined on some interval $\left[r_{-}, b\right]$ with $b<$ $\left.r_{-}+\left(r-r_{0}\right) / N_{0}\right]$ have $F$-length smaller than $r-r_{0}$ and cover a neighborhood $\varphi(W)$ of $\varphi(q)$. So, the neighborhood $W \ni q$ is clearly contained in the required ball. The proof for backward balls is analogous.

Definition 3.10. A conic pseudo-Finsler metric $F$ is (Riemannianly) lower bounded if there exists a Riemannian metric $g_{0}$ on $M$ such that $F(v) \geq \sqrt{g_{0}(v, v)}$ for every $v \in A$. In this case, $g_{0}$ is called a (Riemannian) lower bound of $F$.

Remark 3.11. Several comments are in order:

(1) The lower boundedness needs to be checked only locally, that is, a conic pseudo-Finsler metric $F$ is lower bounded if and only if for every point $p \in M$, there exists a neighborhood $U \subset M$ of $p$ and a Riemannian metric $g$ on $U$ such that $F(v) \geq \sqrt{g(v, v)}$ for every $v \in A \cap T U$. In fact, each $U$ can be chosen compact and, then, all the metrics $g$ can be taken homothetic to a fix auxiliary one $g_{R}$ defined on all $M$. By using paracompactness, a locally finite 
open covering $\left\{U_{i}\right\}_{i \in I}$ of $M$ exists such that $F(v) \geq \frac{1}{c_{i}} \sqrt{g_{R}(v, v)}$ for some $c_{i} \geq 1$ and all $v \in A \cap T U_{i}$. If $\left\{\mu_{i}\right\}_{i \in I}$ is a partition of the unity subordinated to $\left\{U_{i}\right\}_{i \in I}$, then $g_{0}=\frac{1}{\sum_{i \in I} \mu_{i} c_{i}} g$ is the required lower bound of $F$.

(2) The lower boundedness of $F$ can be also checked by looking at the indicatrix $S_{p} \subset A \cap T_{p} M, p \in M$ for each $F_{p}$. Indeed, $F$ is lower bounded if and only iffor each $p \in M$ there exists a compact neighborhood $\hat{K}$ of $0 \in T_{p} M$ in $T M$ such that $S_{q} \subset \hat{K}$ for all $q \in K:=\pi(\hat{K})$.

(3) Either from the definition or from the criteria above, it is straightforward to check that classical Kropina and Matsumoto metrics (see Subsection 4.2 for their definition) are lower bounded.

(4) Observe that the distance $d_{g_{0}}$ associated to the lower bound $g_{0}$ satisfies $d_{g_{0}}(p, q) \leq d_{F}(p, q)$ for every $p, q \in M$ and, then, $B_{F}^{+}(p, \epsilon) \subseteq B_{g_{0}}(p, \epsilon)$.

Lemma 3.12. Let $F: A \rightarrow[0,+\infty)$ be a conic pseudo-Finsler metric in $M$ that admits a lower bound $g_{0}$. Fix $p \in M$ and $r>0$. Given $x \in B_{g_{0}}(p, r)$, there exist $q, q^{\prime} \in B_{g_{0}}(p, r)$ and $\epsilon>0$ such that $x \in B_{F}^{+}(q, \epsilon) \cap B_{F}^{-}\left(q^{\prime}, \epsilon\right)$ and $B_{F}^{+}(q, \epsilon) \cup$ $B_{F}^{-}\left(q^{\prime}, \epsilon\right) \subseteq B_{g_{0}}(p, r)$.

Proof. Consider an admissible curve $\gamma$ passing through $x$. Clearly, we can choose a point $q$ on $\gamma$ such that $d_{F}(q, x)<\epsilon$ and $B_{g_{0}}(q, \epsilon) \subset B_{g_{0}}(p, r)$. So (recall part (4) of Remark 3.11), $x \in B_{F}^{+}(q, \epsilon) \subseteq B_{g_{0}}(q, \epsilon) \subseteq B_{g_{0}}(p, r)$. By reversing the parametrization of $\gamma$, the required point $q^{\prime}$ can be found analogously.

Proposition 3.13. Let $F$ be a lower bounded conic pseudo-Finsler metric on $M$. Then the collection of subsets $\left\{B_{F}^{+}(p, r): p \in M, r>0\right\}$ (respectively $\left\{B_{F}^{-}(p, r)\right.$ : $p \in M, r>0\}$ ) constitute a topological basis of $M$.

Proof. This is a direct consequence of Proposition 3.9 and Lemma 3.12.

\subsection{Discussion on balls and distances}

In general, the Finslerian separation may behave in a very different way from a distance. To make more precise this statement, let us introduce the following notion, see [9, Section 1] and also [54, page 5] and [15].

Definition 3.14. A generalized distance $d$ on a set $X$ is a map $d: X \times X \rightarrow \mathbb{R}$ satisfying the following axioms:

(a1) $d(x, y) \geq 0$ for all $x, y \in X$.

(a2) $d(x, y)=d(y, x)=0$ if and only if $x=y$.

(a3) $d(x, z) \leq d(x, y)+d(y, z)$ for all $x, y, z \in X$.

(a4) Given a sequence $\left\{x_{n}\right\} \subset X$ and $x \in X$, then $\lim _{n \rightarrow \infty} d\left(x_{n}, x\right)=0$ if and only if $\lim _{n \rightarrow \infty} d\left(x, x_{n}\right)=0$.

For any generalized distance, the forward balls have a natural meaning, and generate a topology which coincides with the one generated by the backward balls. Recall 
that the Finslerian distance associated to a Finsler manifold is a generalized distance in the above sense. This can be extended to pseudo-Finsler manifolds, as they are Riemannianly lower bounded.

Theorem 3.15. For any pseudo-Finsler manifold $(M, F)$, the Finslerian separation $d_{F}$ is a generalized distance, the forward (respectively, backward) open balls generate the topology of the manifold, and $d_{F}$ is continuous under this topology.

Proof. Consistency of the definition, including the axiom (a1) in Definition 3.14 follows from part (iii) of Proposition 3.7, and the axiom (a3) from Equation (3.4). For the other two axioms, choose two auxiliary Riemannian metrics $g_{1}, g_{2}$ with distances, respectively, $d_{1}, d_{2}$ which satisfy $g_{1}(v, v) \leq F^{2}(v) \leq g_{2}(v, v)$ for all $v$ in $T M$ (easily, $g_{1}$ and $g_{2}$ can be chosen conformal to any prescribed Riemannian metric $\left.g_{R}\right)$. Obviously:

$$
d_{1} \leq d_{F} \leq d_{2}
$$

From this equality, (a2) and (a4) plus the assertion of the topology of the manifold, follow directly. The last assertion is general for the topology associated to any generalized distance [54, page 6].

Remark 3.16. However, if a vector space $V$ endowed with a pseudo-norm $\|$ • $\|$ is regarded as a pseudo-Finsler space, the affine and $d_{F}$-balls may differ. More precisely, the unit (forward) affine ball is always contained in the corresponding unit (forward) $d_{F}$-one, and the inclusion is strict if $g^{p}$ is indefinite at some $p \in V \backslash\{0\}$.

Theorem 3.15 shows that some properties of $d_{F}$ in the Finsler case are retained in the pseudo-Finsler one. However, in the general conic case the things may be very different, as only Proposition 3.9 can be claimed. As the following example suggests, the relation $\prec$ may give a tidier information.

Example 3.17. (1) In Example 2.23, regarded as a conic pseudo-Finsler manifold $(M, F)$, the following properties occur:

$$
\mathrm{I}^{+}(0)=\{(x, y):|x|<y\}, \quad d_{F}(0, v)= \begin{cases}0 & \text { if } v \in \mathrm{I}^{+}(0), \\ \infty & \text { if } v \notin \mathrm{I}^{+}(0) .\end{cases}
$$

In particular, $d_{F}(0,0)=\infty$ and the open $d_{F}$-balls look very different to the open affine balls.

(2) Moreover, one can obtain a quotient conic pseudo-Finsler metric on the torus $T^{2}=\mathbb{R}^{2} / \mathbb{Z}^{2}$ just taking into account that $F$ is invariant under translations. In this quotient, the Finslerian separation between any two points is equal to 0 .

(3) Regarding Example 2.22 as a conic Finsler metric (with non pointwise convex $A$ ), one also has $d_{F}((0,0),(0,-s))=0$ for all $s \geq 0$ (recall that such $(0,-s)$ does not belong to $A_{(0,0)}$ but $(0,0) \prec(0,-s)$ as one can connect these two points by means of $F$-admissible piecewise smooth curves with a break). Thus, $\{(0,-s): s \geq 0\} \subset B_{F}^{+}((0,0), r)$ but $\{(0,-s): s \geq 0\} \cap B_{(0,0)}^{+}(r)=\emptyset$ for all $r>0$. 
Let us see that even in the conic Finsler case, the Finslerian separation can be discontinuous around two points $p, q$ with $d_{F}(p, q)<\infty$.

Example 3.18. Consider the translation $\varphi: \mathbb{R}^{2} \rightarrow \mathbb{R}^{2}$ given by $\varphi(x, y)=(x+$ $2, y)$ and the group of isometries $G=\left\{\varphi^{n}: n \in \mathbb{Z}\right\}$. The quotient $\mathbb{R}^{2} / G$ can be identified with the strip $\left\{(x, y) \in \mathbb{R}^{2}:-1 \leq x \leq 1\right.$ and $(-1, y) \equiv(1, y)$ $\forall y \in \mathbb{R}\}$. Now consider the convex open cone of $T_{(0,0)} \mathbb{R}^{2}$ determined by the vectors $(-1,3)$ and $(1,1)$ and choose in every point of the cylinder the open cone obtained as the parallel traslation of this cone. This defines the subset $A$. The Finsler metric $F$ is the square root of the Euclidean metric restricted to $A$. Finally observe that the separation from $p=(0,0)$ is not continuous in $q=(-2 / 3,2)$. In fact, $d_{F}((0,0),(-2 / 3,2))=2 \sqrt{13} / 3$ (which is equal to the Euclidean distance from $(0,0)$ to $(4 / 3,2))$, while $d_{F}((0,0),(-2 / 3+\epsilon, 2))=\sqrt{(\epsilon-2 / 3)^{2}+4}$ (the Euclidean distance between the two points) for $0<\epsilon<2$.

\subsection{Minimization properties of geodesics in conic Finsler metrics}

For any conic pseudo-Finsler metric we can define the energy functional as

$$
E_{F}(\alpha)=\int_{a}^{b} F(\dot{\alpha}(t))^{2} \mathrm{~d} s,
$$

where $\alpha:[a, b] \rightarrow M$ is any $F$-admissible piecewise smooth curve between two fixed points $p, q \in M$. Then, geodesics can be defined as critical points of this functional. Of course, they will be also critical points of the length funcional in (3.2), but as critical points of the energy functional, geodesics are obliged to have constant speed.

When the fundamental tensor $g$ is non-degenerate we can define the Chern connection (see [5, Chapter 2]), which is a connection for $\tilde{\pi}^{*}: \pi^{*}(T M) \rightarrow A \backslash\{0\}$ (recall the diagram in (3.1)). Moreover, fixing a vector field $W$ on an open $U \subset M$, the Chern connection gives an affine connection on $U$ that we will denote by $\nabla^{W}$. If we fix a vector field $T$ along a curve $\gamma$, the Chern connection provides a covariant derivative along $\gamma$ with reference $T$ that we will denote as $D^{T}$ (see [44, Sections 7.2 and 7.3]). In this case, geodesics are uniquely determined when the initial conditions are given as the solutions of the equation $D_{T}^{T} T=0$, where $T=\dot{\gamma}$, and there is a unique geodesic tangent to a given vector of the tangent bundle. Moreover, we can define the exponential map in $p \in M$, $\exp _{p}: \Omega \subseteq A_{p}\left(\subseteq T_{p} M\right) \rightarrow M$, as $\exp _{p}(v)=\gamma(1)$, where $\gamma$ is the unique geodesic such that $\gamma(0)=p$ and $\dot{\gamma}(0)=v$, whenever $\gamma$ is defined at least in $[0,1]$.

Convention 3.19. According to our conventions, if $0 \notin A_{p}$, the constant curve $\gamma_{p}(t)=p$ for all $t \in \mathbb{R}$ is not an admissible curve and, so, it is not a geodesic (this situation is common in our study, as it happens in some points whenever a conic pseudo-Finsler metric is not pseudo-Finsler). Nevertheless, when considering any curve $c:[a, b] \rightarrow M$ starting at $p$, we will not care about whether this initial point is obtained from the exponential. So, we will say that $c$ is contained in the image 
by $\exp _{p}$ of some subset $S \subseteq \Omega\left(\subseteq A_{p}\right)$ as the geodesic balls below (or that $c$ lies in $\left.\exp _{p}(S)\right)$, if $c((a, b]) \subset \exp _{p}(S)$, (that is, we will work as if $\exp _{p}(0)=p$ when forced by the context) and we will assume that $\dot{c}(a) \in A_{p}$ (otherwise, the curve would not be admissible).

When the fundamental tensor is degenerate, the interpretation of geodesics as solutions of $D_{T}^{T} T=0$ makes no sense, but as critical points of the energy functional still holds. However, then geodesics are not univocally determined by its velocity at one point (see [32, Example 3 (Figure 1)]; as a limit case, all the constant-speed parameterized curves in the second case of Example 2.21 would be geodesics). Furthermore, if the fundamental tensor is not degenerate but indefinite at some $v \in A_{p}$, the geodesic with velocity $v$ is never minimizing. Next, we will see that for conic Finsler metrics, geodesics minimize in geodesics balls, but a previous technical discussion is required.

Remark 3.20. The Gauss Lemma in typical references such as [5, Lemma 6.1.1] is proven only for standard Finsler metrics. Nevertheless, its local nature allows one to prove it in a much more general context: it works for any conic pseudo-Finsler metric whenever the exponential is defined in a neighborhood $\mathcal{W}$ of $v \in A_{p} \subset T_{p} M$ and $g$ is non-degenerate in $\mathcal{W}$. In fact, put $r=F(v)(>0)$, let $\gamma$ be a geodesic with $\dot{\gamma}(0)=v$, and $w$, a tangent vector to the sphere $S_{0}^{+}(r)=\left\{u \in A_{p}: F(u)=r\right\}$. Choose a curve $\rho:(-\epsilon, \epsilon) \rightarrow S_{0}^{+}(r) \cap \mathcal{W} \subset A_{p}$ such that $\dot{\rho}(0)=w$, and consider the variation $(-\epsilon, \epsilon) \times[0, r] \rightarrow A_{p},(s, t) \mapsto t \rho(s)$. Accordingly, the variation of $\gamma\left(=\gamma_{0}\right)$ by the geodesics $\gamma_{s}(t)=\exp _{p}(t \rho(s))$ with variational field $U$ satisfies

$$
\left.\frac{\partial}{\partial S} \ell_{F}\left(\gamma_{s}\right)\right|_{s=0}=\left.\frac{1}{F(T)} g_{T}(U, T)\right|_{0} ^{r}-\int_{0}^{r} g_{T}\left(U, D_{T}^{T}(T / F(T))\right) \mathrm{d} t,
$$

where $T=\dot{\gamma}$ (see [5, Exercise 5.2.4]). As $\gamma$ is a geodesic, and all the curves in the variation have the same length and depart from the same point, the last equation reduces to the Gauss Lemma, i.e.: $g_{T}\left(d \exp _{p}[w], T\right)=0$.

Proposition 3.21. Let $(M, F)$ be a conic Finsler metric with pointwise convex $A$, and assume that $\exp _{p}$ is defined on a certain ball $B_{0}^{+}(r)$ of $\left(T_{p} M, F_{p}\right)$ and it is a diffeomorphism onto the geodesic ball $B_{p}^{+}(r):=\exp _{p}\left(B_{0}^{+}(r)\right)$. Then, for any $q \in B_{p}^{+}(r)$ the radial geodesic from $p$ to $q$ is, up to reparametrizations, the unique minimizer of the Finslerian separation among the admissible curves contained in $B_{p}^{+}(r)$.

Proof. Given a point $q \in B_{p}^{+}(r)$, let $\tilde{r}$ be the length of the radial geodesic from $p$ to $q$. Consider any smooth admissible curve $c:[0,1] \rightarrow M$ from $p$ to $q$ contained in $B_{p}^{+}(r)$. As $\exp _{p}$ is a diffemorphism on $B_{0}^{+}(r)$, there exist functions $s:[0,1] \rightarrow$ $[0, r)$ and $v:[0,1] \rightarrow S_{0}^{+}(1) \subset T_{p} M$ uniquely determined (both smooth up to zero and $s$ continuous at zero) such that $c(u)=\exp _{p}(s(u) v(u))$ so that $s(0)=0$ and $s(1)=\tilde{r}$. Then, define $\sigma:[0, r) \times[0,1] \rightarrow M$ as $\sigma(t, u)=\exp _{p}(t v(u))$ and 
denote $T=\frac{\partial \sigma}{\partial t}$ and $U=\frac{\partial \sigma}{\partial u}=d \exp _{p}[t \dot{v}]$. We can express $c(u)=\sigma(s(u), u)$ for every $u \in[0,1]$. Hence by the chain rule

$$
\dot{c}(u)=\dot{s}(u) T+U .
$$

Moreover, by the Fundamental Inequality in $(2.8), g_{T}(T, \dot{c}) \leq F(T) F(\dot{c})$, and using the previous identity we get

$$
\dot{s} g_{T}(T, T)+g_{T}(T, U) \leq F(T) F(\dot{c}), \quad \forall s \in(0,1] .
$$

By Gauss Lemma (see Remark 3.20 above), $g_{T}(T, U)=0$, and $F(T)=1$ because $T$ is the velocity of a geodesic with initial velocity equal to $v(u)$ (recall that $F(v(u))=1)$. Moreover, $g_{T}(T, T)=F(T)^{2}=1$ and, therefore, $\dot{s} \leq F(\dot{c})$. Applying the last inequality, we deduce that

$$
\ell_{F}(c)=\int_{0}^{1} F(\dot{c}) d u \geq \lim _{\epsilon \searrow 0} \int_{\epsilon}^{1} \dot{s}(u) d u=s(1)-s(0)=\tilde{r} .
$$

As the length of the radial geodesic is exactly $\tilde{r}$, this shows that it is a global minimizer between all the curves lying in $B_{p}^{+}(r)$.

For the unicity up to reparametrization, observe that the equality in the Fundamental Inequality can happen just when $T$ and $\dot{c}$ are proportional, but looking at Eq. (3.5), this means that $U=0$ (recall that, by Gauss Lemma, $U$ is $g_{T}$-orthogonal to $T)$. As $\exp _{p}$ is a diffeomorphism, $U=0$ implies that $\dot{v}=0$, and therefore, that $c$ is a reparametrization of a radial geodesic.

Remark 3.22. Regarding the absolute minimization:

(1) In the standard Finsler case, the radial geodesic is an absolute minimizer for the curves from $p$ to $q$ on all $M$. In fact, if we consider a curve $\beta$ that goes out of $B_{p}^{+}(r)$, then its length must also be greater or equal than $r$, as the portion of the curve in $B_{p}^{+}(r)$ from $p$ to the boundary of $B_{p}^{+}(r)$ has already length greater or equal to $r$. Summing up, in a Finsler metric, each radial geodesic segment of a geodesic ball as in Proposition 3.21 is the unique curve in $M$ of minimum length which connects its endpoints, up to affine reparameterizations.

(2) This absolute minimization cannot be ensured in the conic Finsler case. The reason is that shorter curves which cross the boundary of $\exp _{p}\left(A_{p}\right)$ may appear. In fact, consider the following example on $\mathbb{R}^{2}$. Let $p=(0,0), q=$ $(0,1) \in \mathbb{R}^{2}$ and $R_{\epsilon}$ the open rectangle of vertexes $V_{p}^{ \pm}(\epsilon)=p+( \pm \epsilon,-\epsilon)$, $V_{q}^{ \pm}(\epsilon)=q+( \pm \epsilon, \epsilon)$ and choose small $\epsilon_{1}>\epsilon_{0}>0$. Consider a Riemannian metric $g=\Lambda\langle\cdot, \cdot\rangle$ (conformal to the usual one $\langle\cdot, \cdot\rangle$ ) such that the conformal factor $\Lambda(>0)$ is equal to some big constant $B$ on $R_{\epsilon_{0}}$ and to 1 outside $R_{\epsilon_{1}} . B$ is chosen so that there are curves $y \mapsto(x(y), y)$ from $p$ to $q$ which go outside $R_{\epsilon_{1}}$ and have $g$-length much smaller than $B$ (which is the $g$-length of the segment from $p$ to $q$ ). Now, let $F$ be the conic Finsler metric 
associated to $g$ with domain $A$ determined as follows. $A_{(x, y)} \equiv(x, y)+C_{y}$ where $C_{y}$ is: (a) the open cone delimited by $p$ and the vertexes $V_{q}^{ \pm}\left(\epsilon_{o}\right)$ for $(x, y)$ with $y \leq 0$, (b) the half plane $y>0$ for $(x, y)$ with $y \geq \delta$ where $\delta \geq 0$ is some prescribed small constant, and (c) a cone obtained by opening the one in (a) until the half plane in (b) for $0<y<\delta$. (Notice that the choice $\delta=0$ is permitted, and then the case (c) will not occur, however, the possibility to choose $\delta>0$ stresses that a choice of $C_{y}$ discontinuous on $y$, is irrelevant here). Then, clearly $B_{p}^{+}\left(1+\epsilon_{0}\right) \subset R_{\epsilon_{0}}$ but there are admissible curves from $p$ to $q$ shorter than the geodesic segment in the ball.

(3) As a consequence of (2), some additional hypothesis must be imposed in order to ensure the character of absolute minimizer for radial geodesics. Taking into account (1), a sufficient hypothesis would be: the boundary of $B_{p}^{+}(r)$ in $\mathrm{I}^{+}(p)$ is equal to $\exp _{p}\left(S_{0}^{+}(r)\right)$.

Remark 3.23. Regarding the domain:

(1) In Proposition 3.21 we have assumed that the domain A is pointwise convex. Even though this could be weakened (only the fundamental inequality was required, and this holds under more general hypotheses, see Remark 2.9), if A is not pointwise convex, some other pathologies may happen. For example, for the conic Finsler norm of Example 2.22, the separation from $(0,0)$ to any point of the form $(0,-s), s \geq 0$ is 0 , but there is no geodesic joining them (recall part (3) of Example 3.17). The underlying reason is that these points $(0,-s)$ lie in $\mathrm{I}^{+}(0,0)$ but not in the affine ball $B_{0}^{+}(r)$ for any $r \geq 0$.

(2) In contrast with the behavior of Example 2.22, Proposition 3.21 plus part (3) of Remark 3.23 imply: in a vector space $V$ endowed with a conic Minkowski norm defined on a convex domain $A$, the $d_{F}$-balls of the Finslerian separation coincide with the affine balls. Indeed, the convexity of each $A_{p}=A \cap T_{p} V$ implies now that $\exp _{p}\left(A_{p}\right)$ coincides with $\mathrm{I}^{+}(p)$.

(3) As a further improvement, to find general conditions on a conic Finsler metric $F$ so that Proposition 3.21 can be applied for small enough affine balls would be very interesting. Proposition 3.13 suggests that the lower boundedness of $F$ might be such a property. Recall that when the Finsler metric is not lower bounded, the forward balls may not constitute a topological basis (see Example 2.22 and the first one in 2.21), and it is not difficult to find cases where the exponential is not defined even in small balls: consider the first Example 2.21 (notice that $A$ is pointwise convex in this case), remove from $\mathbb{R}^{2}$ the semiaxis $\{(x, 0): x \leq 0\}$ and consider any ball $B_{\left(x_{0}, y_{0}\right)}^{+}(r), r>0$ for $\left(x_{0}, y_{0}\right)$ with $y_{0}<0$.

Other properties of local minimization and conjugate points of geodesics in the conic Finsler case deserve to be studied, although we will not go through them. 


\subsection{Summary on geodesics and distance}

Let us summarize and compare in this subsection the properties of geodesics and distance of the different types of Finsler metric generalizations.

Recall first the following three well-known properties of a Finsler manifold:

(i) $d_{F}$ is a generalized distance, $d_{F}$ is continuous, and the open forward (respectively backward) balls generate the topology of $M$.

(ii) The equation of the geodesics is well defined and characterize them univocally from initial data (the velocity at a point). Moreover, the radial geodesic segments of a geodesic ball are strict global minimizers of the energy functional (see part (1) of Remark 3.22).

(iii) In particular, for a Minkowski norm the affine balls and the $d_{F}$-balls agree, and the geodesics as a Finsler manifold coincide with affinely parametrized straight lines for a Minkowski norm.

Let us analyze how these three properties behave in the generalizations of Finsler metrics we are studying.

For a pseudo-Finsler non-Finsler manifold:

(1) Properties in (i) hold (see Theorem 3.15).

(2) Properties in (ii) do not hold. In fact, the equation of the geodesics is necessarily ill defined (as $g^{(p)}$ must be degenerate at some directions, see part (5) of Remark 3.2). Moreover, even in the directions where this equation is well defined, geodesics do not minimize in any sense in general (recall, for example, Remark 3.16). The definition of geodesics as critical curves of the energy functional makes sense, but their uniqueness from the initial velocity does not hold in general (second Example 2.21).

(3) The first assertion in Property (iii) does not hold for Minkowski pseudo-norms whenever $g$ is indefinite (see Remark 3.16). A straightforward computation shows that the affine lines are geodesics for any pseudo-norm, but additional critical curves of the energy functional may appear - in the case of norms, the uniqueness of the affine lines as geodesics hold if the strict triangle inequality hold.

For a conic Finsler manifold:

(1) The two first assertions in Property (i) do not hold in general, as $d_{F}$ may reach the value $\infty$ and also can be discontinuous in finite values (see Example 3.18). The open forward balls do not constitute a basis for the topology in general (first Example 2.21 and 2.22) even though they do in particular cases (Proposition 3.13).

(2) The first assertion in Property (ii) holds, in the sense that the equation of the geodesics is always well defined along the admisible directions. The radial geodesic segments minimize inside geodesic balls (see Proposition 3.21 and also Remark 3.22, especially its part (2)). 
(3) Properties in (iii) hold essentially, but some subtleties must be taken into account. For a conic Minkowski norm with convex $A$ the affine and $d_{F}$ balls coincide (see part (2), and also (1), in Remark 3.23). The affinely parametrized straight lines with velocity in $A$ coincide with the geodesics as a conic Finsler manifold.

For a conic pseudo-Finsler manifold in general the previous properties do not hold, but Propositions 3.9 and 3.13 and relation $\prec$ may yield some general information, which may be useful combined with particular properties of different classes of examples.

\section{Constructing conic Finsler metrics}

New, let us consider the case when a new conic pseudo-Finsler metric is constructed from a homogeneous combination of pre-existing conic Finsler metrics and oneforms, as a generalization (in several senses) of the known $(\alpha, \beta)$-metrics.

\subsection{General result and first consequences}

In the following, $F_{1}, \ldots, F_{n}$ will denote conic Finsler metrics on the manifold $M$ of dimension $N$, with fundamental tensors $g^{1}, \ldots, g^{n}$ (so that $g_{v}^{k}$ is the fundamental tensor of $F_{k}$ at the tangent vector $v$ ). Moreover, the so-called angular metrics (see [5, Equation 3.10.1]) are defined as

$$
h_{v}^{k}(w, w)=g_{v}^{k}(w, w)-\frac{1}{F_{k}(v)^{2}} g_{v}^{k}(v, w)^{2}
$$

for any $v \in A \backslash 0$ and $w \in T_{\pi(v)} M$ and $k=1, \ldots, n$. Due to Cauchy-Schwarz inequality, the angular metric of a conic Finsler metric is always positive semidefinite being the direction of $v$ the only degenerate direction.

The intersection

$$
A=\cap_{k=1}^{n} A_{k} \subset T M
$$

of their conic domains $A_{k}$ is assumed to be non-empty at each point, i.e., $\pi(A)=$ $M$. Moreover, $\beta_{n+1}, \beta_{n+2}, \ldots, \beta_{n+m}$ will denote $m$ one-forms on $M$. The indexes $k, l$ will run from 1 to $n$. The indexes which label the ordering of one-forms will be denoted with Greek letters $\mu, v$ and run from $n+1$ to $n+m$, while the indexes $r, s$ will run from 1 to $n+m$.

Let $B$ be a conic open subset of $\mathbb{R}^{n+m}$ and consider a continuous function $L: B \times M \rightarrow \mathbb{R}$, which satisfies:

(a) $L$ is smooth and positive away from 0 , i.e., on $(B \times M) \backslash(\{0\} \times M)$.

(b) $L$ is $B$-positively homogeneous of degree 2, i.e., $L(\lambda x, p)=\lambda^{2} L(x, p)$ for all $\lambda>0$ and all $(x, p) \in B \times M$. 
Denote by $\operatorname{Hess}(L)$ the $B$-Hessian function matrix associated to $L$, that is, the matrix with coefficients the functions $a_{r s}=L_{, r s}$, where the comma denotes derivative with respect to the corresponding coordinates of $\mathbb{R}^{n+m}$ (in particular, $L_{, r s}$ means the second partial derivative of $L$ with respect to the $r$-th and $s$-th variables). We say that $\operatorname{Hes} s(L)$ is positive semidefinite if so is the matrix of functions at each $(x, p) \in B \times M$. Finally, consider the function $F^{2}: A \subseteq T M \rightarrow \mathbb{R}$ defined as:

$$
F^{2}(v)=L\left(F_{1}(v), F_{2}(v), \ldots, F_{n}(v), \beta_{n+1}(v), \beta_{n+2}(v), \ldots, \beta_{n+m}(v), \pi(v)\right) .
$$

Theorem 4.1. For any $L$ satisfying (a) and (b) as above, and $F^{2}$ as in (4.2), the function $F:=\sqrt{F^{2}}$ is a conic pseudo-Finsler metric with domain $A$ and fundamental tensor:

$$
g_{v}(w, w)=\frac{1}{2} \sum_{k} \frac{L_{, k}}{F_{k}(v)} h_{v}^{k}(w, w)+\frac{1}{2} P(v, w) \operatorname{Hess}(L) P(v, w)^{T},
$$

for all $v \in A \backslash 0, w \in T_{\pi(v)} M$, where the superscript ${ }^{T}$ denotes transpose and $P(v, w)$ the $(n+m)$-tuple

$$
P(v, w)=\left(\frac{g_{v}^{1}(v, w)}{F_{1}(v)}, \ldots, \frac{g_{v}^{n}(v, w)}{F_{n}(v)}, \beta_{n+1}(w), \ldots, \beta_{n+m}(w)\right) \quad\left(\in \mathbb{R}^{n+m}\right),
$$

and the derivatives $L_{, r}, L_{, r s}, \ldots$ of $L$ are computed on

$$
\left(F_{1}(v), F_{2}(v), \ldots, F_{n}(v), \beta_{n+1}(v), \beta_{n+2}(v), \ldots, \beta_{n+m}(v), \pi(v)\right) \in B \times M .
$$

Moreover, $g$ is positive semi-definite if the following conditions hold on the points in (4.5) obtained by taking any $v \in A \backslash 0$ :

(A) $L_{, k} \geq 0$ for every $k=1, \ldots, n$, and

(B) $\operatorname{Hess}(L)$ is positive semi-definite,

and it is positive definite (i.e., $F$ is a conic Finsler metric) if, additionally:

(C) $L_{, 1}+\ldots+L_{, n}>0$.

Proof. Recall from Proposition 2.2 that $g_{v}(v, w)=\left.\frac{1}{2} \frac{\partial}{\partial s} F(v+s w)^{2}\right|_{s=0}$. As, clearly,

$$
\left.\frac{\partial F_{k}(v+s w)}{\partial s}\right|_{s=0}=\left.\frac{\partial \sqrt{F_{k}(v+s w)^{2}}}{\partial s}\right|_{s=0}=\frac{1}{F_{k}(v)} g_{v}^{k}(v, w)
$$

and $\left.\frac{\partial \beta_{\mu}(v+s w)}{\partial s}\right|_{s=0}=\beta_{\mu}(w)$, we have

$$
2 g_{v}(v, w)=\left.\frac{\partial F^{2}(v+s w)}{\partial s}\right|_{s=0}=\sum_{k} \frac{1}{F_{k}(v)} g_{v}^{k}(v, w) L_{, k}+\sum_{\mu} \beta_{\mu}(w) L_{, \mu} .
$$


Again from Proposition 2.2,

$g_{v}(w, w)=\left.\frac{1}{2} \frac{\partial}{\partial t}\right|_{t=0}\left(\left.\frac{\partial}{\partial s} F^{2}((v+t w)+s w)\right|_{s=0}\right)=\left.\frac{\partial}{\partial t} g_{v+t w}(v+t w, w)\right|_{t=0}$

and, then, applying (4.6), we obtain

$$
\begin{aligned}
2 g_{v}(w, w)= & \sum_{k} \frac{L_{, k}}{F_{k}(v)}\left(g_{v}^{k}(w, w)-\frac{1}{F_{k}(v)^{2}} g_{v}^{k}(v, w)^{2}\right) \\
& +\sum_{k, l} \frac{L_{, k l}}{F_{k}(v) F_{l}(v)} g_{v}^{k}(v, w) g_{v}^{l}(v, w) \\
& +2 \sum_{k, \mu} \frac{L_{, k \mu}}{F_{k}(v)} g_{v}^{k}(v, w) \beta_{\mu}(w)+\sum_{\mu, v} L_{, \mu v} \beta_{\mu}(w) \beta_{v}(w) .
\end{aligned}
$$

So, the expression of $g_{v}$ in (4.3) follows by using $F_{k}(v)^{2}=g_{v}^{k}(v, v)$, formula (4.1), and that the two last lines of the formula above can be written as $P(v, w) \operatorname{Hess}(L) P(v, w)^{T}$ for $P(v, w)$ as in (4.4).

For its positive semi-definiteness, recall that applying hypothesis (A), plus the fact that the angular metrics $h_{v}^{k}$ in (4.1) are positive semi-definite:

$$
Q_{1}(v, w):=\sum_{k} \frac{L_{, k}}{F_{k}(v)} h_{v}^{k}(w, w) \geq 0
$$

So, the result follows by applying (B) to obtain:

$$
2 g_{v}(w, w)-Q_{1}(v, w)=P(v, w) \operatorname{Hess}(L) P(v, w)^{T} \geq 0
$$

Finally, for the strict positiveness of $g$, if $w=\lambda v$, with $\lambda \neq 0$, then $g_{v}(w, w)=$ $\lambda^{2} F^{2}(v)>0$ and, otherwise, $Q_{1}(v, w)>0$ by hypothesis (C) and because $h_{v}^{k}$ is degenerate only in $v$.

Remark 4.2. Observe that the expression of the fundamental tensor in (4.8) still holds when $F_{1}, \ldots, F_{n}$ are just conic pseudo-Finsler metrics. Indeed, we can obtain more accurate conditions to construct Finsler metrics. For example, we can consider conic pseudo-Finsler metrics with positive semi-definite fundamental tensor satisfying $(A)$ and $(B)$ and to check that for every $w \in T_{\pi(v)} M, v \in A \backslash 0$, there is some term strictly positive.

Next, let us consider two particular cases. The first one is very elementary, and we include it because, as far as we know, it does not appear in the classic books on the subject. 
Corollary 4.3. Let $F_{1}, F_{2}, \ldots, F_{n}$ be conic Finsler metrics on $M$ defined on the same conic domain A. Then $F=F_{1}+F_{2}+\cdots+F_{n}$ is also a conic Finsler metric and its fundamental tensor is given as

$$
g_{v}(w, w)=F(v) \sum_{k} \frac{h_{v}^{k}(w, w)}{F_{k}(v)}+\left(\sum_{k} \frac{g_{v}^{k}(v, w)}{F_{k}(v)}\right)^{2},
$$

for any $v \in A \backslash 0$ and $w \in T_{\pi(v)} M$.

Proof. Apply Theorem 4.1 to $L: \mathbb{R}^{n} \times M \rightarrow \mathbb{R}$, defined as $L\left(\left(x_{1}, \ldots, x_{n}\right), p\right)=$ $\left(\sum_{r} x_{r}\right)^{2}$ (recall $L_{, k}=2\left(\sum_{r} x_{r}\right)$, thus $\left.L_{, k l}=2\right)$, and apply Equation (4.3).

As a generalization to be compared with the results on Randers metrics below:

Corollary 4.4. Consider $n$ conic Finsler metrics and $m$ one-forms as above. Then,

$$
R(v):=\left(\sum_{k=1}^{n} F_{k}(v)^{q}+\sum_{\mu=k+1}^{m+n}\left|\beta_{\mu}(v)\right|^{q}\right)^{\frac{1}{q}}
$$

is a conic pseudo-Finsler metric with domain A if $q \geq 2$, and with domain

$$
A_{R}:=A \backslash\left\{v \in T M: \beta_{\mu}(v)=0 \text { for some } \mu=m+1, \ldots, m+n\right\},
$$

for $2>q \geq 1$. At each case, the fundamental tensor $g$ is given by:

$$
\begin{aligned}
& R(v)^{2 q-2} g_{v}(w, w)=R(v)^{q} \sum_{k} F_{k}(v)^{q-2} h_{v}^{k}(w, w) \\
& +\frac{1}{2}(q-1) \sum_{k, l}\left(F_{k}(v) F_{l}(v)\right)^{q}\left(\frac{g_{v}^{k}(v, w)}{F_{k}(v)^{2}}-\frac{g_{v}^{l}(v, w)}{F_{l}(v)^{2}}\right)^{2} \\
& +\frac{1}{2}(q-1) \sum_{\mu, v}\left|\beta_{\mu}(v) \beta_{v}(v)\right|^{q}\left(\frac{\beta_{\mu}(w)}{\beta_{\mu}(v)}-\frac{\beta_{v}(w)}{\beta_{v}(v)}\right)^{2} \\
& +(q-1) \sum_{k, \mu}\left|F_{k}(v) \beta_{\mu}(v)\right|^{q}\left(\frac{g_{v}^{k}(v, w)}{F_{k}(v)^{2}}-\frac{\beta_{\mu}(w)}{\beta_{\mu}(v)}\right)^{2} \\
& +\left(\sum_{k} F_{k}(v)^{q-2} g_{v}^{k}(v, w)+\sum_{\mu}\left|\beta_{\mu}(v)\right|^{q-2} \beta_{\mu}(v) \beta_{\mu}(w)\right)^{2},
\end{aligned}
$$

for all $v \neq 0$ in the conic domain and $w \in T_{\pi(v)} M$.

As a consequence, $g$ is always positive semi-definite and, if $n \geq 1, R$ is a conic Finsler metric. 
Proof. Consider the function $L: B \times M \rightarrow \mathbb{R}$ with

$$
B=\mathbb{R}^{n+m} \backslash\left\{\left(x_{1}, \ldots, x_{n+m}\right): x_{r}=0 \text { for some } r=1, \ldots, n+m\right\}
$$

defined as

$$
L\left(\left(a_{1}, a_{2}, \ldots, a_{n+m}\right), p\right)=\sqrt[q]{\left(\left|a_{1}\right|^{q}+\left|a_{2}\right|^{q}+\cdots+\left|a_{n+m}\right|^{q}\right)^{2}} .
$$

Let us denote $U=\left|a_{1}\right|^{q}+\left|a_{2}\right|^{q}+\cdots+\left|a_{n+m}\right|^{q}$. Then $L_{, r}=2 a_{r}\left|a_{r}\right|^{q-2} U^{\frac{2}{q}-1}$ and

$$
L_{, r s}=2 \delta_{r s}(q-1)\left|a_{r}\right|^{q-2} U^{\frac{2}{q}-1}+2(2-q) a_{r} a_{s}\left|a_{r} a_{s}\right|^{q-2} U^{\frac{2}{q}-2},
$$

where $\delta_{r s}$ is Kronecker's delta. Observe that if $x=\left(x_{1}, x_{2}, \ldots, x_{n+m}\right) \in \mathbb{R}^{n+m}$, $x \operatorname{Hess}(L) x^{T}=(q-1) U^{\frac{2}{q}-2} \sum_{r, s}\left|a_{r} a_{s}\right|^{q}\left(\frac{x_{r}}{a_{r}}-\frac{x_{s}}{a_{s}}\right)^{2}+2 U^{\frac{2}{q}-2}\left(\sum_{r} x_{r} a_{r}\left|a_{r}\right|^{q-2}\right)^{2}$.

The expression of the fundamental tensor (4.9) follows easily by using the last identity to compute (4.3) - and, then, the other assertions follow directly.

Remark 4.5. The last corollaries can be useful in different situations. For example, let $F$ be a conic Finsler metric on some open subset $U$ of $M$, and $C$ a closed subset of $M$ included in $U$. If $\tilde{F}$ is any conic Finsler metric on $M \backslash C$, by using a partition of the unity, there exists a conic Finsler metric $F^{*}$ defined on all $M$ which extends $F$ on $C$ and $\tilde{F}$ on $M \backslash U$ (compare with Proposition 3.3 and Remark 3.4). The following corollary develops a different application for the isometry group $\operatorname{Iso}(M, F)$ of a Finsler manifold.

Corollary 4.6. Let $F$ be a non-reversible Finsler metric. Then

$$
\tilde{F}(v)=F(v)+F(-v), \quad \hat{F}(v)=\sqrt{F(v)^{2}+F(-v)^{2}},
$$

for $v \in T M$, are reversible Finsler metrics and:

$$
\operatorname{Iso}(M, F) \subseteq \operatorname{Iso}(M, \tilde{F}) \cap \operatorname{Iso}(M, \hat{F}) .
$$

Moreover, this inclusion becomes an equality for the connected components of the identity of each side.

Proof. The inclusion $\operatorname{Iso}(M, F) \subseteq \operatorname{Iso}(M, \tilde{F}) \cap \operatorname{Iso}(M, \hat{F})$ is straightforward. Clearly, it also holds for the connected components of the identity and, for the converse, let $\phi$ belong to the connected component of the right hand side. Recall that

$$
F(v)=\frac{1}{2}\left(\tilde{F}(v) \pm \sqrt{2 \hat{F}(v)^{2}-\tilde{F}(v)^{2}}\right)
$$


with positive sign if $F(v)-F(-v) \geq 0$ and with negative sign otherwise. Both expressions at right hand (with both signs) are $\phi$-invariant, and the radicand of the root is equal to $(F(v)-F(-v))^{2}$. Therefore, $|F(d \phi(v))-F(-d \phi(v))|=\mid F(v)-$ $F(-v) \mid$ for all $v$, and the equality holds even if the absolute values are removed, as $\phi$ belongs to the connected part of the identity. Thus, $F(d \phi(v))=F(v)$ is a consequence of the expression above for $F$ (applied on $v$ and $d \phi(v)$ ) corresponding to the sign of $F(v)-F(-v)$.

Remark 4.7. (1) Notice that the strict inclusion can hold. In fact, for any nonreversible Minkowski norm $\|\cdot\| \equiv F$ on a vector space $V$, minus the identity belongs to $(\operatorname{Iso}(V, \tilde{F}) \cap \operatorname{Iso}(V, \hat{F})) \backslash \operatorname{Iso}(V, F)$.

(2) Corollary 4.6 reduces the proof that the group of isometries of any Finsler metric is a Lie group, to the reversible case (simplifying, say, the proof in [14, Theorem 3.2], which modifies Myers-Steenrod [35] technique for -symmetric- distances). An alternative proof has been also developed recently in [33, Section 2] by proving that $\operatorname{Iso}(M, F)$ is always a closed subgroup of the isometry group of a suitable average Riemannian metric, see also [16].

\subsection{The case of $\left(F_{0}, \beta\right)$ metrics}

Next, we will focus on the application of Theorem 4.1 on just one Finsler metric $F_{0}$ with conic domain $A_{0} \subseteq T M$ and one 1-form $\beta$. A very important case is that of $(\alpha, \beta)$-metrics introduced by M. Matsumoto in [24] (see also [11, 18, $23,26,29-31,49])$ as a generalization of Randers, Kropina and Matsumoto metrics. Here $\alpha$ denotes the square root of a Riemannian metric and $\beta$ a one-form on $M$. In our general setting, we will consider combinations of the form $F_{0}+\beta$, $F_{0}^{2} / \beta$ and $\frac{F_{0}^{2}}{F_{0}-\beta}$, which generalize the Randers, Kropina and Matsumoto $(\alpha, \beta)$ cases (compare also with [17]). Such combinations were named $\beta$-changes in [48] but, in concordance with our approach, they will be called here $\left(F_{0}, \beta\right)$-metrics, that is, a conic pseudo-Finsler metric $F$ is an $\left(F_{0}, \beta\right)$-metric if it is obtained as a positively homogeneous combination of a conic Finsler metric $F_{0}$ and a oneform $\beta$.

In order to characterize the conic domains for the strong convexity of an $\left(F_{0}, \beta\right)$-metric, we will give first a necessary condition.

Proposition 4.8. Assume that the $\left(F_{0}, \beta\right)$-metric $F=\sqrt{L\left(F_{0}(v), \beta(v), x\right)}$ is a conic Finsler metric and the dimension of $M$ is $N>2$. Then $L_{, 1}>0$.

Proof. Let $v \in A \backslash 0$, and denote as $z \in T_{\pi(v)} M$ the $g_{v}^{0}$-dual of $\beta$, i.e. $\beta(w)=$ $g_{v}^{0}(w, z)$ for all $w \in T_{\pi(v)} M$. Consider an orthonormal basis $e_{1}, \ldots, e_{N}$ for $g_{v}^{0}$ such that $e_{1}=v / F_{0}(v)$ and $z=\lambda^{1} e_{1}+\lambda^{2} e_{2}$ for some $\lambda^{1}, \lambda^{2} \geq 0$. Putting 
$w=\sum_{i=1}^{N} w^{i} e_{i}$ and using (4.3):

$$
\begin{aligned}
2 g_{v}(w, w)= & \frac{1}{F_{0}} L_{, 1} \sum_{i=2}^{N}\left(w^{i}\right)^{2}+L_{, 11}\left(w^{1}\right)^{2}+2 L_{, 12}\left(\lambda^{1} w^{1}+\lambda^{2} w^{2}\right) w^{1} \\
& +L_{, 22}\left(\lambda^{1} w^{1}+\lambda^{2} w^{2}\right)^{2}
\end{aligned}
$$

and the result follows by choosing $w=e_{3}$.

Remark 4.9. Rewriting the terms in (4.10) as $a\left(w^{1}\right)^{2}+2 b w^{1} w^{2}+c\left(w^{2}\right)^{2}+$ $d \sum_{i \geq 3}\left(w^{i}\right)^{2}$ the metric $g_{v}$ is positive definite if and only if $d>0, c>0$ and $a c-b^{2}>0$, i.e., $L_{, 1}>0,\left(\lambda^{2}\right)^{2} L_{, 22}+\frac{1}{F} L_{, 1}>0$ and

$$
\left(L_{, 11}+2 \lambda^{1} L_{, 12}+\left(\lambda^{1}\right)^{2} L_{, 22}\right)\left(L_{, 22}\left(\lambda^{2}\right)^{2}+\frac{1}{F_{0}} L_{, 1}\right)-\left(\lambda^{2}\right)^{2}\left(L_{, 12}+\lambda^{1} L_{, 22}\right)^{2}>0 .
$$

For $N=2$ the term in $d$ does not appear, and the condition $L_{, 1}>0$ is dropped.

4.2.1. Canonical form $F=F_{0} \cdot \phi\left(\beta / F_{0}\right)$

Next, for any $\left(F_{0}, \beta\right)$-metric $F=\sqrt{L\left(F_{0}, \beta\right)}$ we define $\phi(s)=\sqrt{L(1, s)}$ and, thus, $F=F_{0} \cdot \phi\left(\beta / F_{0}\right)$. This change yieds convenient expressions for $\left(F_{0}, \beta\right)$-metrics, including the Kropina, Matsumoto and Randers ones. The next result is related to one by Chern and Shen in [12], which is discussed as an Appendix (Subsection 4.3).

Proposition 4.10. Let $\phi: I \subseteq \mathbb{R} \rightarrow \mathbb{R}$ be a smooth function satisfying $\phi>0$. Given a conic Finsler metric $F_{0}: A_{0} \subseteq T M \rightarrow \mathbb{R}$ and a one-form $\beta$, consider the conic pseudo-Finsler metric

$$
F(v)=F_{0}(v) \phi\left(\frac{\beta(v)}{F_{0}(v)}\right)
$$

on $A:=\left\{v \in A_{0}: \beta(v) / F_{0}(v) \in I\right\}$ with the convention $0_{p} \in A_{p}(\subset A)$ if and only if $T_{p} M \backslash\left\{0_{p}\right\} \subset A_{p}$, for each $p \in M$. Put $\psi(s):=\phi^{2}(s)$ for all $s \in I$ and define the functions:

$$
\varphi_{1}:=2 \psi-s \dot{\psi}(=2 \phi(\phi-s \dot{\phi})), \quad \varphi_{2}:=2 \psi \ddot{\psi}-\dot{\psi}^{2}\left(=4 \ddot{\phi} \phi^{3}\right) .
$$

The fundamental tensor $g$ of $F$ is determined by

$$
\begin{aligned}
2 g_{v}(w, w)= & \varphi_{1}\left(\frac{\beta(v)}{F_{0}(v)}\right) h_{v}^{0}(w, w) \\
& +\frac{1}{2} \psi\left(\frac{\beta(v)}{F_{0}(v)}\right)^{-1} \varphi_{2}\left(\frac{\beta(v)}{F_{0}(v)}\right)\left(\frac{\beta(v)}{F_{0}(v)^{2}} g_{v}^{0}(v, w)-\beta(w)\right)^{2} \\
& +\frac{1}{2} \psi\left(\frac{\beta(v)}{F_{0}(v)}\right)^{-1}\left(\varphi_{1}\left(\frac{\beta(v)}{F_{0}(v)}\right) \frac{g_{v}^{0}(v, w)}{F_{0}(v)}+\dot{\psi}\left(\frac{\beta(v)}{F_{0}(v)}\right) \beta(w)\right)^{2},
\end{aligned}
$$

where $h_{v}^{0}$ is the $F_{0}$-angular metric defined in (4.1), $v \in A \backslash 0$ and $w \in T_{\pi(v)} M$. 
Moreover, $F$ is a conic Finsler metric on A if

$$
\begin{aligned}
\varphi_{1} & =2 \psi-s \dot{\psi}>0(\text { i.e. } \phi-s \dot{\phi}>0) \\
\text { and } \quad \varphi_{2} & =2 \psi(s) \ddot{\psi}(s)-\dot{\psi}(s)^{2} \geq 0(\text { i.e. } \ddot{\phi} \geq 0) .
\end{aligned}
$$

Proof. To compute $g$, put $L(a, b)=a^{2} \psi(b / a)$ defined in

$$
\left\{(a, b) \in \mathbb{R}^{2} \backslash\{(0, s): s \in \mathbb{R}\}: b / a \in I\right\} .
$$

Then,

$$
\begin{aligned}
L_{, 1}(a, b) & =2 a \psi(b / a)-b \dot{\psi}(b / a), \\
L_{, 11}(a, b) & =2 \psi(b / a)-2 \frac{b}{a} \dot{\psi}(b / a)+\frac{b^{2}}{a^{2}} \ddot{\psi}(b / a), \\
L_{, 12}(a, b) & =\dot{\psi}(b / a)-\frac{b}{a} \ddot{\psi}(b / a), \\
L_{, 22}(a, b) & =\ddot{\psi}(b / a) .
\end{aligned}
$$

The expression of $g_{v}$ follows easily from (4.3) and the expressions for $L_{, 1}, L_{, r s}$ above, recalling that

$$
\begin{aligned}
\left(\begin{array}{ll}
x_{1} & x_{2}
\end{array}\right) \operatorname{Hess}(\mathrm{L})\left(\begin{array}{l}
x_{1} \\
x_{2}
\end{array}\right)= & \frac{2 \psi(s) \ddot{\psi}(s)-\dot{\psi}(s)^{2}}{2 \psi(s)}\left(s x_{1}-x_{2}\right)^{2} \\
& +\frac{1}{2 \psi(s)}\left((2 \psi(s)-s \dot{\psi}(s)) x_{1}+\dot{\psi}(s) x_{2}\right)^{2},
\end{aligned}
$$

where $s=b / a$ and $x_{1}, x_{2} \in \mathbb{R}$. The positive definiteness of $g_{v}$ under conditions (4.13) is immediate from expression (4.12).

Remark 4.11. In the set of sufficient conditions $\varphi_{1}>0, \varphi \geq 0$ (Equation (4.13)), the first one is also necessary (to obtain the positive definiteness of $g$ ) when $N>2$, as $L_{, 1}\left(F_{0}(v), \beta(v)\right)=F_{0}(v) \varphi_{1}(s)$ for $s=\beta(v) / F(v)$ (recall Proposition 4.8).

\subsubsection{Kropina type metrics}

Kropina metrics are $(\alpha, \beta)$-metrics of the form $\alpha^{2} / \beta$, which were first studied by Kropina in [20] (see also [28,47,51,53]). In our extension, we consider not only an $\left(F_{0}, \beta\right)$-metric but also introduce an arbitrary exponent $q>0$. We remark that in the paper [50], the authors study when two Finsler metrics $F$ and $F_{0}$ are related by $F=F_{0}^{2} / \beta$ for some one-form $\beta$ and in [8, formula (6)], the authors consider a 2-homogeneous Finsler metric of this type.

Corollary 4.12. Consider $F=F_{0}^{q+1} /|\beta|^{q}$ and $A=\left\{v \in A_{0}: \beta(v) \neq 0\right\}$. Then $F$ is a conic Finsler metric defined on A for every $q>0$, with fundamental tensor 
determined by

$$
\begin{aligned}
\left|\frac{\beta(v)}{F_{0}(v)}\right|^{2 q} g_{v}(w, w)= & (q+1) h_{v}^{0}(w, w) \\
& +q(q+1)\left(\frac{g_{v}^{0}(v, w)}{F_{0}(v)}-\frac{F_{0}(v)}{\beta(v)} \beta(w)\right)^{2} \\
& +\left((q+1) \frac{g_{v}^{0}(v, w)}{F_{0}(v)}-q \frac{F_{0}(v)}{\beta(v)} \beta(w)\right)^{2}
\end{aligned}
$$

where $v \in A$ and $w \in T_{\pi(v)} M$. In particular, any Kropina metric $F=\alpha^{2} /|\beta|$ is strongly convex in its natural domain of definition.

Proof. Observe that $F=F_{0}^{q+1} /|\beta|^{q}=F_{0}\left|F_{0} / \beta\right|^{q}$. Now if $\phi(s)=|s|^{-q}, s \in$ $\mathbb{R} \backslash\{0\}$, then $F=F_{0} \phi\left(\beta / F_{0}\right)$. Moreover, inequalities (4.13) become

$$
\varphi_{1}(s)=2(q+1)|s|^{-2 q}>0, \quad \varphi_{2}(s)=4 q(q+1)|s|^{-4 q-2} \geq 0,
$$

which hold whenever $q>0$. Therefore, the formula for $g_{v}$ follows from (4.12) (use $\left.|s|^{2 q} \psi^{-1}(s)=|s|^{4 q} ; \dot{\psi}(s)=-2 q|s|^{-2 q} s^{-1}\right)$, and positive definiteness is obvious from the expression of $g_{v}$.

\subsubsection{Matsumoto type metrics}

Matsumoto metrics were found by this author when measuring the walking time when there is a slope [27]. They are $(\alpha, \beta)$-metrics of the form $\alpha^{2} /(\alpha-\beta)$. We extend them in the same way as Kropina's.

Corollary 4.13. For any $q \neq 0$, consider the conic pseudo-Finsler metric:

$$
F=F_{0}^{q+1} /\left|F_{0}-\beta\right|^{q}, \quad A=\left\{v \in A_{0}: F_{0}(v) \neq \beta(v)\right\} .
$$

Then, its fundamental tensor is determined by

$$
\begin{aligned}
\left|\frac{F_{0}(v)-\beta(v)}{F_{0}(v)}\right|^{2 q+2} g_{v}(w, w)= & \frac{\left(F_{0}(v)-\beta(v)\right)\left(F_{0}(v)-(q+1) \beta(v)\right)}{F_{0}(v)^{2}} h_{v}^{0}(w, w) \\
& +q(q+1)\left(\frac{\beta(v)}{F_{0}(v)^{2}} g_{v}^{0}(v, w)-\beta(w)\right)^{2} \\
& +\left(\frac{F_{0}(v)-(q+1) \beta(v)}{F_{0}(v)^{2}} g_{v}^{0}(v, w)+q \beta(w)\right)^{2},
\end{aligned}
$$

for any $v \in A$ and $w \in T_{\pi(v)} M$.

When $q>0$ or $q \leq-1$ the restriction of $F$ to

$$
A^{*}=\left\{v \in A_{0}:\left(F_{0}(v)-(q+1) \beta(v)\right)\left(F_{0}(v)-\beta(v)\right)>0\right\}
$$

is conic Finsler and, if $N>2$, $g$ is not strongly convex at any point of $A \backslash A^{*}$. 
Proof. As $F=F_{0}^{q+1} /\left|F_{0}-\beta\right|^{q}=F_{0}\left(F_{0} /\left|F_{0}-\beta\right|\right)^{q}$, putting $\phi(s)=|1-s|^{-q}$, $s \neq 1$, then $F=F_{0} \phi\left(\beta / F_{0}\right)$. So, inequalities (4.13) read

$$
\begin{aligned}
& \varphi_{1}(s)=2|1-s|^{-2 q-2}(1-s)(1-(1+q) s)>0, \\
& \varphi_{2}(s)=4 q(q+1)|1-s|^{-4 q-2} \geq 0,
\end{aligned}
$$

which hold if and only if $(1-s)(1-(1+q) s)>0$ and either $q \geq 0$ or $q \leq-1$. So, the formula for $g_{v}$ follows from (4.12) (use $\psi(s)^{-1} \varphi_{2}(s)=4 q(q+1) / / 1-$ $\left.\left.s\right|^{2 q+2} ; \dot{\psi}(s)=-2 q|1-s|^{-2 q}(1-s)^{-1}\right)$, and strong convexity in $A^{*}$ from the expression of $g_{v}$. For the last assertion, apply Remark 4.11 noticing that $A^{*}$ determines the region where $L_{, 1}>0$.

Remark 4.14. (1) In the case $N=2$, the maximal domain $A^{*}$ where such a generalized Matsumoto metric is a conic Finsler one, becomes more involved (see Remark 4.9). As a particular case of Corollary 4.25 in the Appendix, the following necessary and sufficient condition to define $A^{*}$ is obtained (for simplicity, we put $r=1)$ :

$$
3 \beta(v)<2\|\beta\|_{g_{v}^{0}}^{2}+1 \quad \text { for any } v \in A \text { with } F_{0}(v)=1 .
$$

(2) The class of metrics of the last corollary with $F_{0}=\alpha$ and $r<-1$, were considered in [55] in order to obtain Finsler metrics with constant flag curvature.

In particular, a known result on Matsumoto metric is recovered.

Corollary 4.15. Any Matsumoto metric $F=\alpha^{2} /|\alpha-\beta|$ is strongly convex in

$$
A^{*}=\left\{v \in A_{0}:(\alpha(v)-2 \beta(v))(\alpha(v)-\beta(v))>0\right\} .
$$

As a last consequence, we consider a class of metrics which include those in the references $[13,21,46]$.

Corollary 4.16. Let us define $F=\left(F_{0}+\beta\right)^{2} / F_{0}$ and

$$
A=\left\{v \in A_{0}: F_{0}(v)^{2}>\beta(v)^{2}\right\} .
$$

Then $F$ is strongly convex in A.

Proof. Apply Corollary 4.13 with $q=-2$.

\subsubsection{Randers type metrics}

Randers metrics are $(\alpha, \beta)$-metrics defined by $\alpha+\beta$ (they are named after the work of G. Randers [39] about electromagnetism). Next we will consider Finsler metrics of the form $F_{0}+\beta$, which generalize Randers construction. In particular our result can be used to prove strong convexity of Randers metric in a more direct way than in [5, page 284]. Moreover, it follows that the deformations of Kropina metrics considered in [40] are also strongly convex. 
Corollary 4.17. Any metric $F=F_{0}+\beta$, where $A=\left\{v \in A_{0}: F_{0}(v)+\beta(v)>0\right\}$, is a conic Finsler metric on all A with fundamental tensor

$$
g_{v}(w, w)=\frac{F_{0}(v)+\beta(v)}{F_{0}(v)} h_{v}^{0}(w, w)+\left(\frac{g_{v}^{0}(v, w)}{F_{0}(v)}+\beta(w)\right)^{2}
$$

for any $v \in A \backslash 0$ and $w \in T_{\pi(v)} M$.

Proof. Put $F=F_{0}\left(1+\frac{\beta}{F_{0}}\right)$ and define $\phi(s)=1+s$. As $\varphi_{2} \equiv 0$, inequalities (4.13) reduce to $\varphi_{1}=2(1+s)>0$, and the proof follows from Proposition 4.10.

Notice that, essentially, this result can be also regarded as a particular case of Corollary 4.4 .

4.2.5. A further generalization: $\left(F_{1}, F_{2}\right)$-metrics

From now on, $F_{1}$ and $F_{2}$ will be two conic Finsler metrics defined in a common conic domain $A_{0}$. In order to show the power of our computations, let us explore the consequences of changing $\beta$ into a second Finsler metric in $\left(F_{0}, \beta\right)$-metrics.

Proposition 4.18. Let $\phi: I \subset \mathbb{R} \rightarrow \mathbb{R}, \phi>0$ be a smooth function, and put $\psi\left(=\phi^{2}\right), \varphi_{1}, \varphi_{2}$ as in Proposition 4.10. Define the conic pseudo-Finsler metric:

$$
F(v)=F_{1}(v) \phi\left(\frac{F_{2}(v)}{F_{1}(v)}\right)
$$

on $A=\left\{v \in A_{0}: F_{2}(v) / F_{1}(v) \in I\right\}$, with the convention $0_{p} \in A_{p}(\subset A)$ if and only if $T_{p} M \backslash\left\{0_{p}\right\} \subset A_{p}$, for each $p \in M$. The fundamental tensor $g$ of $F$ is:

$$
\begin{aligned}
& 2 g_{v}(w, w)=\varphi_{1}\left(\frac{F_{2}(v)}{F_{1}(v)}\right) h_{v}^{1}(w, w)+\frac{F_{1}(v)}{F_{2}(v)} \dot{\psi}\left(\frac{F_{2}(v)}{F_{1}(v)}\right) h_{v}^{2}(w, w) \\
& +\frac{1}{2} \psi\left(\frac{F_{2}(v)}{F_{1}(v)}\right)^{-1} \varphi_{2}\left(\frac{F_{2}(v)}{F_{1}(v)}\right)\left(\frac{F_{2}(v)}{F_{1}(v)^{2}} g_{v}^{1}(v, w)-\frac{g_{v}^{2}(v, w)}{F_{2}(v)}\right)^{2} \\
& \quad+\frac{1}{2} \psi\left(\frac{F_{2}(v)}{F_{1}(v)}\right)^{-1}\left(\varphi_{1}\left(\frac{F_{2}(v)}{F_{1}(v)}\right) \frac{g_{v}^{1}(v, w)}{F_{1}(v)}+\dot{\psi}\left(\frac{F_{2}(v)}{F_{1}(v)}\right) \frac{g_{v}^{2}(v, w)}{F_{2}(v)}\right)^{2},
\end{aligned}
$$

for any $v \in A \backslash 0$ and $w \in T_{\pi(v)} M$. Moreover, $F$ is a conic Finsler metric on $A$, if $\dot{\psi} \geq 0$ and Equation (4.13) is satisfied (i.e. $\varphi_{1}>0, \varphi_{2} \geq 0$ ).

Proof. The proof is analogous to that of Proposition 4.10. Just observe that $L_{, 2}(a, b)=a \dot{\psi}(b / a)$ and apply Theorem 4.1 as in Proposition 4.10. 
Corollary 4.19. For any $q \neq 0$, consider the conic pseudo-Finsler metric:

$$
F=F_{1}^{q+1} /\left|F_{1}-F_{2}\right|^{q}, \quad A=\left\{v \in A_{0}: F_{1}(v) \neq F_{2}(v)\right\}
$$

Then, its fundamental tensor is given by

$$
\begin{aligned}
& \left(\frac{F_{1}(v)-F_{2}(v)}{F_{1}(v)}\right)^{2 q+2} g_{v}(w, w) \\
& =\frac{\left(F_{1}(v)-F_{2}(v)\right)\left(F_{1}(v)-(q+1) F_{2}(v)\right)}{F_{1}(v)^{2}} h_{v}^{1}(w, w) \\
& \quad+q \frac{F_{1}(v)\left(F_{1}(v)-F_{2}(v)\right)}{F_{2}(v)^{2}} h_{v}^{2}(w, w) \\
& \quad+q(q+1)\left(\frac{F_{2}(v)}{F_{1}(v)^{2}} g_{v}^{1}(v, w)-\frac{g_{v}^{2}(v, w)}{F_{2}(v)}\right)^{2} \\
& \quad+\left(\frac{F_{1}(v)-(q+1) F_{2}(v)}{F_{1}(v)^{2}} g_{v}^{1}(v, w)+q \frac{g_{v}^{2}(v, w)}{F_{2}(v)}\right)^{2}
\end{aligned}
$$

where $v \in A$ and $w \in T_{\pi(v)} M$.

When $q>0$ the restriction of $F$ to

$$
A^{*}=\left\{v \in A_{0}: F_{1}(v)-(q+1) F_{2}(v)>0 \quad \text { and } \quad F_{1}(v)-F_{2}(v)>0\right\} \text {, }
$$

is conic Finsler.

Proof. Apply Proposition 4.18 following the same lines as in Corollary 4.13. Notice that we have to ensure now $F_{1}>F_{2}$ in the definition of $A^{*}$ because, otherwise, $\dot{\psi}(s)$ is not positive (recall the third line in the expression of $g_{v}$ ).

\subsection{Appendix}

Let us remark that in [12, Lemma 1.1.2], the authors obtained a result closely related to our Proposition 4.10 for $(\alpha, \beta)$-metrics, concretely:

Criterion 4.20 (Chern and Shen, [12]). $F=\alpha \phi(\beta / \alpha)$ is a Minkowski norm for any Riemannian metric $\alpha$ and 1-form $\beta$ with $\|\beta\|_{\alpha}<b_{0}$ if and only if $\phi=\phi(s)>0$ satisfies the following conditions:

$$
(\phi(s)-s \dot{\phi}(s))+\left(b^{2}-s^{2}\right) \ddot{\phi}(s)>0,
$$

where $s$ and $b$ are arbitrary numbers with $|s| \leq b<b_{0}$, and $\phi$ is defined in a symmetric interval $\left(-b_{0}, b_{0}\right)$. 
Remark 4.21. To compare with Proposition 4.10, recall that there, the hypotheses (4.13) on $\phi$ were $\phi(s)-s \dot{\phi}(s)>0$ and $\ddot{\phi}(s) \geq 0$. Clearly, these two hypotheses imply (4.16). Conversely, (4.16) implies the first of the two hypotheses (choose $b=s$, and notice that any $b \leq\|\beta\|_{\alpha}$ can be chosen), but it is somewhat weaker than the second one. The reason is that the criterion above assumes $\|\beta\|_{\alpha}<b_{0}$ but no assumption on $\beta$ was done in Proposition 4.10. However, even in this case, the criterion can be applied to give our sufficient condition. Namely, taking an increasing sequence of compact neighborhoods $\left\{K_{j}\right\}$ which exhausts $M$, for each $K_{j}$ there exists a constant $b_{j}$ which plays the role of $b_{0}$ and, if $\left\{b_{j}\right\} \rightarrow \infty$ then condition (4.16) splits into the two conditions of Proposition 4.10.

For the sake of completeness, Criterion 4.20 will be reobtained next for $\left(F_{0}, \beta\right)$ metric, and its hypothseses will be stated in a more accurate way (see Remark 4.26). We will follow the approach in previous references on the topic computing the determinant of the matrix $\left(g_{v}\right)_{i j}$ explicitly.

Let us fix a coordinate system $\varphi: U \rightarrow \bar{U} \subset \mathbb{R}^{N}$ and denote $\frac{\partial}{\partial x^{1}}, \ldots, \frac{\partial}{\partial x^{N}}$, the vector fields associated to the system. Moreover, $g_{i j}(v)$ and $g_{i j}^{0}(v)$ will denote respectively the coordinates of the fundamental tensors $g_{v}$ and $g_{v}^{0}$ associated to $F=\sqrt{L\left(F_{0}, \beta\right)}$ and $F_{0}$ respectively. Then from (4.3) it follows that

$$
\begin{aligned}
2 g_{i j}(v)= & \frac{L_{, 1}}{F_{0}}\left(g_{i j}^{0}(v)-\frac{1}{F_{0}(v)^{2}} v_{i} v_{j}\right)+\frac{L_{, 11}}{F_{0}(v)^{2}} v_{i} v_{j} \\
& +\frac{L_{, 12}}{F_{0}(v)}\left(v_{i} b_{j}+v_{j} b_{i}\right)+L_{, 22} b_{i} b_{j},
\end{aligned}
$$

where $v=\sum_{i=1}^{N} v^{i} \frac{\partial}{\partial x^{i}}, v_{i}=\sum_{j=1}^{N} g_{i j}^{0}(v) v^{j}$ and $b_{i}=\omega\left(\frac{\partial}{\partial x^{i}}\right), i=1, \ldots, N$. Our goal is to compute the determinant of the matrix $\left(g_{i j}(v)\right)$. We will need the following algebraic result whose proof can be found in [25, Proposition 30.1] (for symmetric matrices) or in [5, Proposition 11.2.1].

Lemma 4.22. Let $P=\left(p_{i j}\right)$ and $Q=\left(q_{i j}\right)$ be $n \times n$ real matrices and $C=\left(c_{i}\right)$ be an $n$-vector. Assume that $Q$ is invertible with $Q^{-1}=\left(q^{i j}\right)$, and $p_{i j}=q_{i j}+\delta c_{i} c_{j}$. Then

$$
\operatorname{det}\left(p_{i j}\right)=\left(1+\delta c^{2}\right) \operatorname{det}\left(q_{i j}\right),
$$

where $c:=\sqrt{\sum_{i, j=1}^{n} q^{i j} c_{i} c_{j}}$. Therefore, if $1+\delta c^{2} \neq 0$, then $P$ is invertible, and its inverse matrix $P^{-1}=\left(p^{i j}\right)$ is given by

$$
p^{i j}=q^{i j}-\frac{\delta c^{i} c^{j}}{1+\delta c^{2}},
$$

where $c^{i}:=\sum_{j=1}^{n} q^{i j} c_{j}$. 
We remark that in $[41,42]$ similar computations to those of the following lemma have been carried out for $(\alpha, \beta)$-metrics.

Lemma 4.23. Denote $\Delta_{L}=\operatorname{det}(\operatorname{Hess}(L))$. Then

$$
\begin{aligned}
& \operatorname{det}\left(g_{i j}(v)\right) \\
& =\frac{L_{, 1}^{N-2}}{2^{N} F_{0}(v)^{N+1}}\left(F_{0}(v) \Delta_{L}\left(F_{0}(v)^{2}\|\beta\|_{g_{v}^{0}}^{2}-\beta(v)^{2}\right)+2 L_{, 1} L\right) \operatorname{det}\left(g_{i j}^{0}(v)\right) .
\end{aligned}
$$

Proof. First observe that

$$
g_{i j}(v)=\frac{L_{, 1}}{2 F_{0}(v)}\left(g_{i j}^{0}(v)+\delta(v)\left(b_{i}+k(v) v_{i}\right)\left(b_{j}+k(v) v_{j}\right)+\mu(v) v_{i} v_{j}\right),
$$

where

$$
\delta(v)=\frac{L_{, 22} F_{0}(v)}{L_{, 1}}, \quad k(v)=\frac{L_{, 12}}{L_{, 22} F_{0}(v)} \quad \text { and } \quad \mu(v)=\frac{\Delta_{L}}{L_{, 1} L_{, 22} F_{0}(v)}-\frac{1}{F_{0}(v)^{2}} .
$$

If we call $c_{i}=b_{i}+k(v) v_{i}$ and $g^{0}(v)^{i j}=\left(g_{i j}^{0}\right)^{-1}(v)$, applying twice Lemma 4.22, we obtain that

$$
\begin{aligned}
\operatorname{det}\left(g_{i j}(v)\right)= & \frac{L_{, 1}^{N}}{2^{N} F_{0}(v)^{N}}\left(\left(1+\mu(v) \sum_{i, j=1}^{N} g^{0}(v)^{i j} v_{i} v_{j}\right)\left(1+\delta(v) c^{2}\right)\right. \\
& \left.-\mu(v) \delta(v) \sum_{i, j=1}^{N} c^{i} c^{j} v_{i} v_{j}\right) \operatorname{det}\left(g_{i j}^{0}(v)\right)
\end{aligned}
$$

where

$$
\begin{gathered}
c^{2}=\sum_{i, j=1}^{N} g^{0}(v)^{i j} c_{i} c_{j}=\sum_{i, j=1}^{N} g^{0}(v)^{i j}\left(b_{i}+k(v) v_{i}\right)\left(b_{j}+k(v) v_{j}\right) \\
=\|\beta\|_{g_{v}^{0}}^{2}+2 k(v) \beta(v)+k(v)^{2} F_{0}(v)^{2} \\
\sum_{i, j=1}^{N} g^{0}(v)^{i j} v_{i} v_{j}=F_{0}(v)^{2} \text { and } \\
\sum_{i, j=1}^{N} c^{i} c^{j} v_{i} v_{j}=\sum_{i, j=1}^{N}\left(b^{i}+k(v) v^{i}\right)\left(b^{j}+k(v) v^{j}\right) v_{i} v_{j}=\left(\beta(v)+k(v) F_{0}(v)^{2}\right)^{2} .
\end{gathered}
$$


Then, formula (4.18) follows substituting $\delta(v), k(v)$ and $\mu(v)$ by their values, and making some straightforward computations (use

$$
F_{0}(v)^{2} L_{, 11}+\beta(v)^{2} L_{, 22}+2 \beta(v) F_{0}(v) L_{, 12}=2 L,
$$

which follows from evaluating (4.3) at $w=v$ and recalling (2.2)).

Proposition 4.24. If $F=F_{0} \phi\left(\beta / F_{0}\right)$, then $\operatorname{det}\left(g_{i j}(v)\right)$ is equal to

$$
\left(\phi-\frac{\beta(v)}{F_{0}(v)} \dot{\phi}\right)^{N-2}\left(\left(\|\beta\|_{g_{v}^{0}}^{2}-\frac{\beta(v)^{2}}{F_{0}(v)^{2}}\right) \ddot{\phi}+\phi-\frac{\beta(v)}{F_{0}(v)} \dot{\phi}\right) \phi^{N+1} \operatorname{det}\left(g_{i j}^{0}(v)\right) .
$$

Proof. Using the expressions of the partial derivatives of $L$ computed in Proposition 4.10, Equation (4.18) becomes, in terms of $\psi=\phi^{2}$,

$$
\begin{aligned}
\operatorname{det}\left(g_{i j}(v)\right)= & \frac{\left(2 F_{0}(v) \psi-\beta \dot{\psi}\right)^{2}}{2^{N} F_{0}(v)^{N+1}}\left(\left(2 \psi \ddot{\psi}-(\dot{\psi})^{2}\right) F_{0}(v)\left(F_{0}(v)^{2}\|\beta\|_{g_{v}^{0}}^{2}-\beta(v)^{2}\right)\right. \\
& \left.+\left(2 F_{0}(v) \psi-\beta(v) \dot{\psi}\right) 2 \psi F_{0}(v)^{2}\right) .
\end{aligned}
$$

Then substituting $\psi=\phi^{2}, \dot{\psi}=2 \phi \dot{\phi}$ and $\ddot{\psi}=2\left((\dot{\phi})^{2}+\phi \ddot{\phi}\right)$ and making some straightforward computations we obtain (4.19).

Corollary 4.25. Let $F=F_{0} \phi\left(\beta / F_{0}\right)$, choose $v_{0} \in A \backslash 0$ (see Proposition 4.10 ), and put $s_{0}=\beta\left(v_{0}\right) / F_{0}\left(v_{0}\right)$. In the case of dimension $N>2$, the fundamental tensor $g_{v_{0}}$ is positive definite if and only if

$$
\begin{array}{r}
\phi\left(s_{0}\right)-s_{0} \dot{\phi}\left(s_{0}\right)>0, \\
\ddot{\phi}\left(s_{0}\right)\left(\|\beta\|_{g_{v_{0}}^{0}}^{2}-s_{0}^{2}\right)+\phi\left(s_{0}\right)-s_{0} \dot{\phi}\left(s_{0}\right)>0
\end{array}
$$

and, in the case of dimension $N=2, g_{v_{0}}$ is positive definite if and only if the second inequality holds.

Proof. Define $\phi_{t}(s)=1-t+t \phi(s)$, in the same domain as $\phi$ for $t \in[0,1]$. Then, in dimension $N>2$

$$
\phi_{t}\left(s_{0}\right)-s_{0} \dot{\phi}_{t}\left(s_{0}\right)=1-t+t\left(\phi\left(s_{0}\right)-s_{0} \dot{\phi}\left(s_{0}\right)\right)>0
$$


and in any dimension $N \geq 2$

$$
\begin{aligned}
& \ddot{\phi}_{t}\left(s_{0}\right)\left(\|\beta\|_{g_{v_{0}}^{0}}^{2}-s_{0}^{2}\right)+\phi_{t}\left(s_{0}\right)-s_{0} \dot{\phi}_{t}\left(s_{0}\right) \\
& =1-t+t\left[\ddot{\phi}\left(s_{0}\right)\left(\|\beta\|_{g_{v_{0}}^{0}}^{2}-s_{0}^{2}\right)+\phi\left(s_{0}\right)-s_{0} \dot{\phi}\left(s_{0}\right)\right]>0 .
\end{aligned}
$$

Then, let $F_{t}\left(v_{0}\right)=F_{0}\left(v_{0}\right) \phi_{t}\left(\beta\left(v_{0}\right) / F_{0}\left(v_{0}\right)\right), g_{v_{0}}^{t}$, the fundamental tensor associated to $F_{t}$, and $\left(g_{i j}^{t}\left(v_{0}\right)\right)$ the matrix of coordinates of $g_{v_{0}}^{t}$ in the coordinate chart previously fixed. Applying Proposition 4.24 to each $\phi_{t}$, one has $\operatorname{det}\left(g_{i j}^{t}\left(v_{0}\right)\right)>0$ for every $t \in[0,1]$. Observe that for $t=0, F_{t}=F_{0}$, and, consequently, $\left(g_{i j}^{0}\left(v_{0}\right)\right)$ is positive definite. Then, as $\operatorname{det}\left(g_{i j}^{t}\left(v_{0}\right)\right)>0$ for every $t \in[0,1], g_{i j}^{1}\left(v_{0}\right)$ must be positive definite.

For the converse, observe that Proposition 4.8 implies that $\phi\left(s_{0}\right)-s_{0} \dot{\phi}\left(s_{0}\right)>0$ for $N>2$, and the other inequality follows for any $N \geq 2$ by using (4.19), as $\operatorname{det}\left(g_{i j}\left(v_{0}\right)\right)$ must be positive.

Remark 4.26. The inequalities in the previous corollary characterize in a precise way the maximal conic domain $A^{*}$ where $F$ is conic Finsler. In the second inequality, observe that $\|\beta\|_{g_{v_{0}}}^{2}-s_{0}^{2} \geq 0$ (this follows directly from the definition of the norm $\|\beta\|_{g_{v_{0}}^{0}}$ and the equality $\left.F_{0}\left(v_{0}\right)=\sqrt{g_{v_{0}}^{0}\left(v_{0}, v_{0}\right)}\right)$.

In the case that $F_{0}=\alpha$ (i.e. $F$ is a conic $(\alpha, \beta)$-metric) then $\|\beta\|_{g_{v_{0}}^{0}}$ depends only on the point $p_{0}=\pi\left(v_{0}\right)$ and we write $\|\beta\|_{\alpha_{p_{0}}}$ instead. Moreover, if $F$ is an $(\alpha, \beta)$-metric (with $A=T M$ ), then an $\alpha$-unit vector $v_{0}$ can be chosen both, in the kernel of $\beta$ and such that $\|\beta\|_{\alpha_{p_{0}}}=\beta\left(v_{0}\right)$, and as a consequence, $s^{2}=$ $\beta(v)^{2} / \alpha(v)^{2}, v \in A_{p_{0}} \backslash\{0\}$, takes all the values in the interval $\left[0,\|\beta\|_{\alpha_{p_{0}}}^{2}\right]$ (notice that, in this case, 0 must belong to the domain $I$ of definition of $\phi$ ). Thus, the following criterion (to be compared with Criterion 4.20, taking into account Remark 4.21) is obtained: an $(\alpha, \beta)$-metric $F=\alpha \phi(\beta / \alpha)$ is a Finsler one if and only if the inequalities in Corollary 4.25 hold for all $s \in \mathbb{R}$ such that $0 \leq s^{2} \leq\|\beta\|_{\alpha}:=$ $\operatorname{Sup}_{p \in M}\|\beta\|_{\alpha_{p}}$., i.e.:

$$
\begin{aligned}
\phi(s)-s \dot{\phi}(s) & >0, \\
\ddot{\phi}(s)\left(\|\beta\|_{\alpha}^{2}-s^{2}\right)+\phi(s)-s \dot{\phi}(s) & >0,
\end{aligned}
$$

the latter under the convention that, if $\|\beta\|_{\alpha}=\infty$ then $\ddot{\phi} \geq 0$ on $I$.

\section{References}

[1] M. AвAte and G. Patrizio, "Finsler Metrics - a Global Approach. With applications to geometric function theory", Lecture Notes in Mathematics, Vol. 1591, Springer-Verlag, Berlin, 1994. 
[2] H. AKbaR-ZADEh, "Initiation to Global Finslerian Geometry", North-Holland Mathematical Library, Vol. 68, Elsevier Science B.V., Amsterdam, 2006.

[3] P. L. Antonelli, R. S. Ingarden and M. Matsumoto, "The Theory of Sprays and Finsler Spaces with Applications in Physics and Biology", Fundamental Theories of Physics, Vol. 58, Kluwer Academic Publishers Group, Dordrecht, 1993.

[4] G. S. AsAnOv, "Finsler Geometry, Relativity and Gauge Theories", Fundamental Theories of Physics, Vol. 12, D. Reidel Publishing Co., Dordrecht, 1985.

[5] D. BAO, S.-S. CHERN and Z. SHEN, "An Introduction to Riemann-Finsler Geometry", Graduate Texts in Mathematics, Vol. 200, Springer-Verlag, New York, 2000.

[6] R. Bartolo, E. Caponio, A. V. Germinario and M. SÁnchez, Convex domains of Finsler and Riemannian manifolds, Calc. Var. Partial Differential Equations 40 (2011), 335356.

[7] A. Bejancu and H. R. FARran, "Geometry of Pseudo-Finsler Submanifolds", Kluwer Academic Publishers, Dordrecht, 2000.

[8] G. Y. Bogoslovsky and H. F. Goenner, Finslerian spaces possessing local relativistic symmetry, Gen. Relativity Gravitation 31 (1999), 1565-1603.

[9] H. Busemann, Local metric geometry, Trans. Amer. Math. Soc. 56 (1944), 200-274.

[10] R. L. BRYANT, Some remarks on Finsler manifolds with constant flag curvature, Houston J. Math. 28 (2002), 221-262.

[11] I. BUCATARU, Nonholonomic frames in Finsler Geometry, Balkan J. Geom. Appl. 7 (2002), $13-27$.

[12] S.-S. CHERN and Z. SHEN, "Riemann-Finsler Geometry", Nankai Tracts in Mathematics, Vol. 6, World Scientific Publishing Co. Pte. Ltd., Hackensack, NJ, 2005.

[13] N. CUI and Y.-B. SHEn, Projective change between two classes of $(\alpha, \beta)$-metrics, Differential Geom. Appl. 27 (2009), 566-573.

[14] S. Deng and Z. Hou, The group of isometries of a Finsler space, Pacific J. Math. 207 (2002), 149-155.

[15] J. L. Flores, J. Herrera and M. SÁnchez, "Gromov, Cauchy and Causal Boundaries for Riemannian, Finslerian and Lorentzian Manifolds", Memoirs Amer. Math. Soc., Vol. 226, 2013.

[16] R. GAlLego TORRomé, Averaged structures associated with a Finsler structure, arXiv: math/0501058v9 [math.DG].

[17] M. Hashiguchi, How to get examples of Finsler spaces, Confer. Sem. Mat. Univ. Bari 225 (1987), 13 pp.

[18] M. Kitayama, M. Azuma and M. Matsumoto, On Finsler spaces with $(\alpha, \beta)$-metric. Regularity, geodesics and main scalars, J. Hokkaido Univ. Ed. Sect. II A 46 (1995), 1-10.

[19] S. KobaYashi and K. Nomizu, "Foundations of Differential Geometry", Vol. I, Wiley Classics Library, John Wiley \& Sons Inc., New York, 1996. Reprint of the 1963 original, A Wiley-Interscience Publication.

[20] V. K. KRopinA, On projective Finsler spaces with a metric of some special form, Naucn. Dokl. Byss. Skoly 2 (1959), 38-42.

[21] B. LI and Z. SHEN, On a class of projectively flat Finsler metrics with constant flag curvature, Internat. J. Math. 18 (2007), 749-760.

[22] R. L. Lovas, A note on Finsler - Minkowski norms, Houston J. Math. 33 (2007), 701-707.

[23] I. M. MASCA, V. S. SABAU and H. ShIMADA, Reversible geodesics for $(\alpha, \beta)$-metrics, Internat. J. Math. 21 (2010), 1071-1094.

[24] M. Matsumoto, On C-reducible Finsler spaces, Tensor (N.S.) 24 (1972), 29-37. Commemoration volumes for Prof. Dr. Akitsugu Kawaguchi's seventieth birthday, Vol. I.

[25] M. Matsumoto, "Foundations of Finsler Geometry and Special Finsler Spaces", Kaiseisha Press, Shigaken, 1986.

[26] M. MAtsumoto, A remarkable connection in a Finsler space with $(\alpha, \beta)$-metric, Tensor (N.S.) 48 (1989), 241-244. 
[27] M. MAтsumoto, A slope of a mountain is a Finsler surface with respect to a time measure, J. Math. Kyoto Univ. 29 (1989), 17-25.

[28] M. Matsumoto, Finsler spaces of constant curvature with Kropina metric, Tensor (N.S.) 50 (1991), 194-201.

[29] M. Matsumoto, Projectively flat Finsler spaces with $(\alpha, \beta)$-metric, Rep. Math. Phys. 30 (1991), 15-20.

[30] M. Matsumoto, A special class of locally Minkowski spaces with $(\alpha, \beta)$-metric and conformally flat Kropina spaces, Tensor (N.S.) 50 (1991), 202-207.

[31] M. Matsumoto, Theory of Finsler spaces with $(\alpha, \beta)$-metric, Rep. Math. Phys. 31 (1992), 43-83.

[32] V. S. MatVeEv, Riemannian metrics having common geodesics with Berwald metrics, Publ. Math. Debrecen 74 (2009), 405-416.

[33] V. S. Matveev, H.-B. Rademacher, M. Troyanov and A. Zeghib, Finsler conformal Lichnerowicz-Obata conjecture, Ann. Inst. Fourier (Grenoble) 59 (2009), 937-949.

[34] X. Mo, "An Introduction to Finsler Geometry", Peking University Series in Mathematics, Vol. 1, World Scientific Publishing Co. Pte. Ltd., Hackensack, NJ, 2006.

[35] S. B. MYERS and N. E. STEENROD, The group of isometries of a Riemannian manifold, Ann. of Math. (2) 40 (1939), 400-416.

[36] B. O'NeILl, "Semi-Riemannian Geometry", with applications to relativity, Vol. 103, Pure and Applied Mathematics, Academic Press Inc. [Harcourt Brace Jovanovich Publishers], New York, 1983.

[37] V. PERLICK, Fermat principle in Finsler spacetimes, Gen. Relativity Gravitation 38 (2006), $365-380$.

[38] C. Pfeifer and M. N. Wohlfarth, Causal structure and electrodynamics on Finsler spacetimes, Phys. Rev. D (4) 84 (2011), 044039-044042.

[39] G. RANDERS, On an asymmetrical metric in the fourspace of general relativity, Phys. Rev. (2) 59 (1941), 195-199.

[40] M. Roman, On a deformation of the Kropina metric, Differ. Geom. Dyn. Syst. 8 (2006), 236-243 (electronic).

[41] V. S. SABAU and H. Shimada, Classes of Finsler spaces with $(\alpha, \beta)$-metrics, Rep. Math. Phys. 47 (2001), 31-48.

[42] V. S. SABAU and H. Shimada, Errata, Rep. Math. Phys. 51 (2003), 149-152.

[43] M. SÁNCHEZ, Geodesic connectedness of semi-Riemannian manifolds, In: "Proceedings of the Third World Congress of Nonlinear Analysts", part 5, Catania, 2000, Vol. 47, 2001, 3085-3102.

[44] Z. SHEN, "Differential Geometry of Spray and Finsler Spaces", Kluwer Academic Publishers, Dordrecht, 2001.

[45] Z. SHEN, "Lectures on Finsler Geometry", World Scientific Publishing Co., Singapore, 2001 .

[46] Z. SHEN and G. CIVI YILDIRIM, On a class of projectively flat metrics with constant flag curvature, Canad. J. Math. 60 (2008), 443-456.

[47] C. ShIBATA, On Finsler spaces with Kropina metric, Rep. Math. Phys. 13 (1978), 117-128.

[48] C. SHIBATA, On invariant tensors of $\beta$-changes of Finsler metrics, J. Math. Kyoto Univ. 24 (1984), 163-188.

[49] H. SHIMADA, S. V. SABAU and R. S. INGARDEN, The $(\alpha, \beta)$-metric for Finsler space and its role in thermodynamics, Bull. Soc. Sci. Lett. Łódź Sér. Rech. Déform. 57 (2008), 25-38.

[50] U. P. Singh, B. N. PRASAD and B. KUMARI, On a Kropina change of Finsler metric, Tensor (N.S.) 64 (2003), 181-188.

[51] U. P. SINGH and A. K. Singh, On conformal transformations of Kropina metric, Period. Math. Hungar. 16 (1985), 187-192.

[52] A. C. Thompson, "Minkowski Geometry", Encyclopedia of Mathematics and its Applications, Vol. 63, Cambridge University Press, Cambridge, 1996. 
[53] R. YoshiKAWA and K. OKUво, Kropina spaces of constant curvature, Tensor (N.S.) 68 (2007), 190-203.

[54] E. M. Zaustinsky, "Spaces with Non-symmetric Distance", Mem. Amer. Math. Soc. No., Vol. 34, 1959.

[55] L. ZHOU, A local classification of a class of $(\alpha, \beta)$ metrics with constant flag curvature, Differential Geom. Appl. 28 (2010), 170-193.

\author{
Departamento de Matemáticas \\ Universidad de Murcia, \\ Campus de Espinardo \\ 30100 Espinardo, Murcia, Spain \\ majava@um.es \\ Departamento de Geometría y Topología \\ Facultad de Ciencias \\ Universidad de Granada \\ Campus Fuentenueva s/n \\ 18071 Granada, Spain \\ sanchezm@ugr.es
}

